\title{
On the Curvature of Piecewise Flat Spaces
}

\author{
Jeff Cheeger ${ }^{1, \star}$, Werner Müller ${ }^{2}$, and Robert Schrader ${ }^{3, \star \star \star \star \star \star}$ \\ 1 Department of Mathematics, SUNY at Stony Brook, Stony Brook, NY 11794, USA \\ 2 Zentralinstitut für Mathematik, Akademie der Wissenschaften der DDR, Mohrenstrasse 39, \\ DDR-1199 Berlin, German Democratic Republic \\ 3 Institute for Theoretical Physics, SUNY at Stony Brook, Stony Brook, NY 11794, USA
}

\begin{abstract}
We consider analogs of the Lipschitz-Killing curvatures of smooth Riemannian manifolds for piecewise flat spaces. In the special case of scalar curvature, the definition is due to T. Regge; considerations in this spirit date back to J. Steiner. We show that if a piecewise flat space approximates a smooth space in a suitable sense, then the corresponding curvatures are close in the sense of measures.
\end{abstract}

\section{Introduction}

Let $X^{n}$ be a complete metric space and $\Sigma \subset X^{n}$ a closed, subset of dimension less than or equal to $(n-1)$. Assume that $X^{n} \backslash \Sigma$ is isometric to a smooth (incomplete) $n$-dimensional Riemannian manifold. How should one define the curvature of $X^{n}$ at points $x \in \Sigma$, near which the metric need not be smooth and $X$ need not be locally homeomorphic to $U \subset R^{n}$ ? In $[\mathrm{C}, \mathrm{Wi}]$, this question is answered (in seemingly different, but in fact, equivalent ways) for the Lipschitz-Killing curvatures, and their associated boundary curvatures under the assumption that the metric on $X^{n}$ is piecewise flat. The precise definitions (which are given in Sect. 2), are formulated in terms of certain "angle defects." For the mean curvature and the scalar curvature they are originally due to Steiner [S] and Regge [R], respectively.

It is worth noting at the outset that the discussion of curvature at nonsmooth points depends in a crucial way on the precise notion of curvature under consideration. If, for example, one wishes to generalize the Pontrjagin forms, the notion of "angle defect" will no longer suffice. It can be replaced by the much less elementary " $\eta$-invariant" (see $[\mathrm{C}]$ ).

Recall that in the smooth case, the $j^{\text {th }}$ Lipschitz-Killing curvature $R^{j}$ is the measure on $M^{n}$, which is zero for $j$ odd and which for $j$ even is given by integrating

\footnotetext{
* Supported in part by NSF MCS-810-2758-A-02

$\star \star$ Supported in part by Deutsche Forschungsgemeinschaft and NSF PHY-81-09110-A-01

$\star \star \star$ On leave of absence from Freie Universität Berlin
} 
the form

$$
\begin{gathered}
R^{j}=\left((n-j) ! 2^{j} \pi^{j / 2}(j / 2) !\right)^{-1}(-1)^{j / 2}, \\
\sum_{\pi}(-1)^{|\pi|} \Omega_{\pi(1) \pi(2)} \wedge \ldots \wedge \Omega_{\pi(j-1) \pi(j)} \wedge \omega_{\pi(j+1)} \wedge \ldots \wedge \omega_{\pi(n)} .
\end{gathered}
$$

Here the summation is over all permutations of $n$ elements. The $\Omega_{k l}$ are the curvature 2-forms, indexed with respect to a locally defined orthonormal frame $e_{k}$ for the tangent bundle $T M^{n}$ of $M^{n}$. The 1 -forms $\omega_{k}$ are the dual basis to $e_{k}$. Thus if $\omega_{k l}$ denote the connection 1-forms, the structure equations take the form

$$
\begin{gathered}
d \omega_{k}=-\sum_{i} \omega_{k l} \wedge \omega_{l}, \\
d \omega_{k l}=-\sum_{i} \omega_{k i} \wedge \omega_{i l}+\Omega_{k l}
\end{gathered}
$$

Similarly, the $j^{\text {th }}$ mean curvature $H^{j}$ is the measure on $\partial M^{n}$, given as follows. Assume that $\left\{e_{k}\right\}$ has been chosen so that along $\partial M^{n} e_{n}$ coincides with (say) the inward normal. For any $2 k+1 \leqq j \leqq n$ set

$$
\begin{aligned}
Q_{j, k}= & c_{j, k} \sum_{\pi}(-1)^{|\pi|} \Omega_{\pi(1) \pi(2)} \wedge \ldots \wedge \Omega_{\pi(2 k-1) \pi(2 k)} \\
& \wedge \omega_{\pi(2 k+1), n} \wedge \ldots \wedge \omega_{\pi(j-1), n} \wedge \omega_{\pi(j)} \wedge \ldots \wedge \omega_{\pi(n-1)},
\end{aligned}
$$

where now $\pi$ runs through all permutations of $n-1$ elements, and where

$$
c_{j, k}= \begin{cases}(-1)^{k}\left(2^{j} \pi^{\frac{j-1}{2}} k !\left(\frac{j-1}{2}-k\right) !(n-j) !\right)^{-1}, & j \text { odd } \\ (-1)^{k}\left(\pi^{j / 2} k ! 2^{k+\frac{j}{2}}(j-2 k-1) !(n-j) !\right), & j \text { even } .\end{cases}
$$

We then put

$$
H^{j}=\sum_{k} Q_{j, k}
$$

The normalization is chosen such that

$$
\int_{T^{n-j \times M^{j}}} R^{j}+\int_{T^{n-j \times \partial M^{j}}} H^{j}=\chi\left(M^{j}\right) \operatorname{Vol}\left(T^{n-j}\right)
$$

for any flat $T^{n-j}$.

In particular $R^{0}$ is the volume, $R^{2}$ is the scalar curvature and $R^{n}$ the ChernGauss-Bonnet form if $n$ is even. Similarly, $H^{1}$ is the area of the boundary, $H^{2}$ the mean curvature for the inward normal, etc. Note that the integrated $R^{j}, H^{j}$ do not depend on a choice of orientation.

Let $R^{c}$ denote a piecewise flat analog of some particular curvature $R$, in the smooth case. Among the properties which $R^{c}$ should enjoy are

1) It should be an invariantly defined local measurement of the intrinsic geometry of $X$ which vanishes if $X^{n}$ is flat.

2) The significance of $R^{c}$ should be analogous to that of $R$. More precisely, consider some formula (e.g. Chern-Gauss-Bonnet) which expresses a certain 
analytic, geometric, or topological measurement of $X$ in terms of $R$. If this measurement still makes sense in the singular case (e.g. the Euler characteristic) then the formula should continue to hold with $R$ replaced by $R^{c}$. Note that condition 2) necessitates further similarities between $R$ and $R^{c}$, for example the same scaling properties and behavior under metric products. Moreover for the Lipschitz-Killing curvatures in general there are at least three formulas to which condition 2) applies: Chern's kinematic formula [Ch], Weyl's tube formula [We], and the expression for the Lipschitz-Killing curvatures in terms of the asymptotics of the heat kernel (see [C, CMS2, Sa, SW] for details) ${ }^{1}$.

We can now state in rough terms the main result of the present work. Let $M^{n}$ be a smooth Riemannian space and let $M_{\eta}^{n}$ be a sequence of piecewise flat spaces which converge to $M^{n}$ in a suitable sense (which entails a certain uniform nondegeneracy on the simplices, see Sect. 1 for precise definitions). Let $R_{\eta}$ and $R$ denote a Lipschitz-Killing curvature of $M_{\eta}^{n}$ and $M^{n}$, respectively, or an associated boundary term. Then we assert that $R_{\eta} \rightarrow R$, where the (local) convergence is in the sense of measures ${ }^{2}$.

To take the most elementary illustration, let $M^{2}$ be a compact region in the plane with smooth boundary. Choose points $\left\{p_{i}\right\} \subset \partial M^{2}$ and form a polygonal approximation $M_{\eta}^{2}$, to $M^{2}$ by connecting $p_{i-1}$ to $p_{i}$ by a segment $\sigma_{i}$. Assume that for all i, $\sigma_{i}$ has length $\eta$. Then the geodesic curvature of $\partial M_{\eta}^{2}$ is concentrated at $\left\{p_{i}\right\}$. By definition, the weight (angle defect) attached to $p_{i}$ is

$$
\pi-\alpha_{i}
$$

where $\alpha_{i}$ is the interior angle between $\sigma_{i}, \sigma_{i+1}$.

We can assume that near $p_{i}, \partial M^{2}$ is given by $y=f(x)$, with $p_{i}$ located at $(0,0)$ and $f^{\prime}(0)=0$. Then as is well known, the geodesic curvature of $\partial M^{2}$ at $p_{i}$ is equal to $f^{\prime \prime}(0)$. Thus, the total curvature of a segment of length $\eta$ centered at $p_{i}$ is

$$
\sim f^{\prime \prime}(0) \cdot \eta
$$

Let $\Theta_{j}$ denote the angle between $\sigma_{j}$ and the $x$ axis, $j=i, i+1$. Then

$$
\Theta_{j} \sim \tan \Theta_{j} \sim \frac{1}{2} f^{\prime \prime}(0) \eta^{2} / \eta
$$

from which it follows that

$$
\pi-\alpha_{i} \sim f^{\prime \prime}(0) \eta
$$

The above example suggests that settling the convergence problem in general, might just amount to calculating the pointwise limit of an appropriate angle defect. But this turns out to be somewhat misleading. In the general case, the pointwise calculation of $\lim _{\eta \rightarrow 0} R_{\eta}^{j}$ results in an expression which merely resembles, but does not

1 Indeed it was by comparing the formulas for the first variation of the surface area for smooth and polyhedral surfaces that Steiner [S] arrived at the definition for the mean curvature in the polyhedral case

2 In the case of mean curvatures of convex hypersurfaces of euclidean space this is contained implicity in the work of Minkowski [Mi]. The assumption of convexity simplifies the situation considerably in this context 
coincide with $R^{j}$. In particular the limit is not independent of the choice of approximating sequence $M_{\eta}^{n}$ (see Appendix 1).

Recall, however, our actual assertion that $R_{\eta} \rightarrow R$ in the sense of measures. This means the following. We fix an open set $U \subset M^{n}$, and then count all contributions corresponding to points lying in $U$. Thus, when the approximation becomes fine, we are counting a large number of small contributions. So the possibility exists that these might give the correct answer on the average, even though they fail to do so individually. Remarkably, this averaging effect does indeed take place, and in this sense, the convergence, $R_{\eta} \rightarrow R$, is not a purely local phenomenon.

We now explain the general strategy behind our proof of convergence, the details of which will be presented in Sects. 5-8. Consider the interior term $R^{j}$. We begin with the observation that it suffices to restrict attention to $U=B_{r}(p)$, a metric ball of radius $r$ centered at $p$, and to let $r \rightarrow 0$ in such a way that $\eta=o(r)$. Our main local calculation shows that the contribution to $R_{\eta}^{j}$ coming from $B_{r}(p)$ is

$$
\sim P_{j}\left(r, T_{\eta}\right) \operatorname{Vol}\left(B_{r}(p)\right) \text {. }
$$

Here $P_{j}$ is a certain polynomial in the curvature at $p$ of degree $j / 2$ which depends on $r$ and the triangulation $T_{\eta}$. The coefficients of this polynomial, however, are uniformly bounded, independent of the particular choice of triangulation (see Sects. 4 and 5).

By duality, $R^{j}$ also defines a polynomial $* R^{j}$ in curvature of degree $j / 2$ :

$$
R^{j}=\left(* R^{j}\right) \omega,
$$

(where $\omega$ is the canonical volume form). According to Gilkey [G1] (see also [D]), among all polynomials of degree $j / 2$ in curvature, $* R^{j}$ is characterized by the following properties ( $E^{k}$ denotes flat euclidean space).

1) It is invariant.

2) It vanishes if the space splits as a metric product $M^{j-1} \times E^{n-j+1}$.

3 ) It agrees with $* R^{j}$ for any curvature tensor at which $R^{j} \neq 0$, e.g. that of

$$
S^{2} \times S^{2} \times \ldots \times S^{2} \times E^{n-j} \quad\left(j / 2 \text { factors of } S^{2}\right) .
$$

There is also a similar characterization of the boundary term $H^{j}$, see [G2].

It is not at all obvious that $P_{j}\left(r, T_{\eta}\right)$ has any of the above properties, nor in particular that it is independent of the choice of triangulation $T_{\eta}$. But conversely, if this independence can be established, the invariance property follows almost. immediately. The vanishing property and normalization can then be verified by considering special approximating triangulations and metrics, which possess product decompositions as in 2) and 3) above.

To establish that $P_{j}\left(r, T_{\eta}\right)$ is independent of $T_{\eta}$, we proceed in two steps.

Step 1. Consider two triangulations $T_{\eta_{1}}$ and $T_{\eta_{2}}$ of $B_{r}(p)$. We will find two other triangulations $T_{\eta_{3}}$ and $T_{\eta_{4}}$ of an open subset $U$ of $B_{r}(p)$, such that

1) $T_{\eta_{3}}$ and $T_{\eta_{4}}$ coincide near the boundary of $U$,

2) $T_{\eta_{3}}$ and $T_{\eta_{4}}$ agree with $T_{\eta_{1}}$ and $T_{\eta_{2}}$, respectively, in the interior up to distances $O\left(\eta_{1}+\eta_{2}\right)$ to the boundary.

Thus for the boundary contributions $H^{j}$ to the Lipschitz-Killing curvatures

$$
H_{\eta_{3}}^{j}=H_{\eta_{4}}^{j},
$$


while by $(0.12)$ the interior terms satisfy

$$
\begin{aligned}
& R_{\eta_{3}}^{j}=R_{\eta_{1}}^{j}+O\left(\frac{\eta_{1}}{r}\right)+O(r), \\
& R_{\eta_{4}}^{j}=R_{\eta_{2}}^{j}+O\left(\frac{\eta_{2}}{r}\right)+O(r) .
\end{aligned}
$$

Step 2. Using a generalization of a variational calculation of Regge (see [R] and Sects. 2 and 3) we show that for the total curvatures

$$
R_{\eta_{3}}^{j}+H_{\eta_{3}}^{j}=R_{\eta_{4}}^{j}+H_{\eta_{4}}^{j}+O\left(\frac{\eta_{3}+\eta_{4}}{r}\right)+O(r) .
$$

Combining (0.14)- $(0.16)$ gives

$$
R_{\eta_{1}}^{j}=R_{\eta_{2}}^{j}+O\left(\frac{\eta_{1}+\eta_{2}+\eta_{3}+\eta_{4}}{r}\right)+O(r),
$$

which suffices to complete the proof. A similar argument establishes the local convergence, $H_{\eta}^{j} \rightarrow H^{j}$, for the boundary curvatures.

We remark that for $j>2, n>2$, it is not clear a priori, that the sequence of measures $R_{\eta}^{j}$ is even bounded. Thus, $(0.12)$ is already quite strong. ${ }^{3}$

We close this introduction with a word about the physical motivation which underlies our work (see also Appendix 3). This pertains primarily to the scalar curvature $R^{2}$ and mean curvature $H^{2}$. As is well known, these curvatures enter into the Hilbert action principle from which the Einstein field equations are derived. Thus, it was with a view towards applications in general relativity that Regge, [R], introduced the scalar curvature of piecewise flat approximations to a Riemannian manifold. In this fundamental work, Regge dealt with the convergence $R_{\eta}^{2} \rightarrow R^{2}$ at the heuristic level but did not attempt a rigorous proof. Nonetheless, convergence seems to have been taken for granted by those physicists who subsequently discussed "Regge calculus."

Although Regge worked in a context which was purely classical, later Wheeler [Wh] speculated on the possibility of employing Regge calculus as a tool for constructing a quantum theory of gravity (see also [CMS1, Frö, H, HP, HS, L2, MTW, PR, RW1, RW2, Wei, Wa]). This approach is in some respects similar to the use of lattice approximations in gauge theories, and thus might also be useful for numerical calculations (for further discussions of Regge calculus in the classical context, see [CW1, CW3, L1, WE, Wo]).

It is amusing to notice that our results immediately imply a new proof of the Chern-Gauss-Bonnet theorem for smooth Riemannian manifolds (related to Gilkey's characterization of the Chern-Gauss-Bonnet form in a manner analogous to the heat equation proof). We point out that Wheeler (perhaps unaware of the work of Allendoerfer and Weil [AW]) also asked for an analog in Regge calculus of the Chern-Gauss-Bonnet form.

3 Relation (0.12) is one of the places where the above mentioned uniform nondegeneracy is used. (Compare also the well known example of H. A. Schwarz; see [Ra, pp. 6-7]) 
The results of this paper pertain to Riemannian manifolds. However, a similar discussion seems possible for pseudo-Riemannian manifolds, by suitably avoiding the light cone directions.

The remainder of the paper is organized into eight sections as follows.

1. Basic Properties of Piecewise Flat Spaces

2. Linear Algebra

3. Lipschitz-Killing Curvatures for Piecewise Flat Spaces

4. Limiting Values of Face Angles

5. Statement of Main Theorem and Strategy of Proof

6. Proof Modulo Lemma on Triangulations

7. Lemma on Triangulations

8. Convergence of Boundary Curvatures

Appendix 1. Local Calculations

Appendix 2. Variable Curvature and Dihedral angles

Appendix 3. Riemannian Metric on the Space of Piecewise Flat Structures

Throughout the paper we make the following convention. We indicate the dependence on parameters of constants appearing in an estimate by writing e.g. $c(n)$ for any constant depending only on $n$. Conversely, if any parameter within the context considered does not appear, it means the constant can be estimated independently of this parameter.

\section{Preliminaries}

For the convenience of some readers and to establish notation, we review the elementary properties of piecewise flat (piecewise linear) spaces.

A finite simplicial complex $K$ consists of a finite set of elements called vertices and a set of finite nonempty subsets of vertices called simplices such that

(1) Any set containing only one vertex is a simplex.

(2) Any nonempty subset of a simplex is also a simplex.

A $j$-simplex will generally be noted by $\sigma^{j}$. The dimension $j$ is the number of its vertices minus 1 . The 1 -simplices are called edges. If $\sigma^{\prime} \subset \sigma, \sigma^{\prime} \neq \sigma, \sigma^{\prime}$ is called a face of $\sigma$. We set $\operatorname{dim} K=\sup _{\sigma \in K} \operatorname{dim} \sigma$ and generally write $K^{n}$. A complex $L$ is called a subcomplex of $K$ if the simplices of $L$ are also simplices of $K$. We write $L \subset K$. The $k$-skeleton, $\Sigma^{k}\left(K^{n}\right)$ of $K^{n}(0 \leqq k \leqq n)$ is the subcomplex formed by the $j$-simplices $(j \leqq k)$ of $K^{n}$.

Let $p=\left\{p_{j}\right\}, 1 \leqq j \leqq q+1$ be points in $E^{n}, n>q$, which lie in no $(q-1)$ dimensional affine subspace. The convex hull, $\bar{\sigma}(p)$ and its interior $\sigma(p)$ are called closed and open linear simplices, respectively. By regarding $p_{j}=v_{j}$ as vectors, we have

$$
\sum_{j} x_{j} v_{j}=\sigma(p)
$$

where $\left\{x_{j}\right\}$ consists of $q+1$-tuples with $x_{j}>0$ and

$$
\sum_{j} x_{j}=1 \text {. }
$$


The barycenteric coordinates $\left\{x_{j}\right\}$ of $q \in \sigma(p)$ are independent of choice of origin in $E^{n}$. A map from $\sigma^{q}(p)$ to $\sigma^{q}\left(p^{\prime}\right)$ which preserves barycentric coordinates is called linear.

If $e_{1}, \ldots, e_{n}$ are the standard basis vectors in $E^{n}$, their convex hull is called the standard (closed) simplex $\sigma(n)$. To any finite simplicial complex with $n$ (ordered) vertices, we associate a closed subset ${ }^{s} K$ of $\sigma(n)$ called the geometric realization of $K$. Namely, to each simplex $\sigma^{i} \in K$ with vertices $\sigma_{j_{1}}^{0}, \ldots, \sigma_{j_{t+1}}^{0}$, we associate the open linear simplex determined by $e_{j_{1}}, \ldots, e_{j_{i+1}}$. The union of these linear simplices is ${ }^{s} K$. There is a natural metric space structure, the standard metric on ${ }^{s} K$, where the distance between two points $p, q$ is defined as the infimum of the length of all piecewise smooth paths between $p$ and $q$. More generally, we consider metrics $\varrho$ on ${ }^{s} K$ such that any simplex $\sigma^{i} C^{s} K$ with its induced metric is linearly isometric to some linear simplex. The space ${ }^{s} K$, equipped with a metric of the above type is called a triangulated piecewise flat space (or p.l. space). Clearly, any such space is determined up to isometry by its edge lengths, the distances between the vertices of 1 -simplices. Thus, we might write $(K, l)$ if we wish to emphasize the dependence on the collection of edge lengths $l$.

A simplicial cone $C^{k} C E^{k} C E^{n}$ is the intersection of $k$ open half spaces $H_{j}$ through the origin, which lie in general position. Alternatively it is the set on which the corresponding linear functionals are all positive. The intersection, $L^{k-1}$, of $C^{k}$ with the unit sphere, is by definition a spherical simplex. We write $C^{k}=C\left(L^{k-1}\right)$, where $C\left(L^{k-1}\right)$ is flat and $L^{k-1}$ carries a metric of constant curvature $\equiv 1$.

Given linear simplices $\sigma^{l} \subset \sigma^{k}$, at any point $q \in \sigma^{l}$, we have the normal cone $C^{\perp}\left(\sigma^{l}, \sigma^{k}\right)$ consisting of all rays through $q$ which are orthogonal to $\sigma^{l}$ and point into $\sigma^{k}$. $C^{\perp}\left(\sigma^{l}, \sigma^{k}\right)$ is well defined up to isometry and independent of $q$. The associated spherical simplex, $L\left(\sigma^{l}, \sigma^{k}\right)$ is called the link of $\sigma^{l}$ in $\sigma^{k}$.

Fig. 1.1

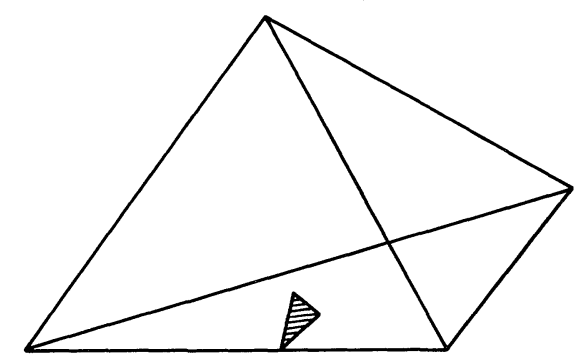

Alternatively, for sufficiently small $\varepsilon, L\left(\sigma^{l}, \sigma^{k}\right)$ can be identified with the set of points $x \in \sigma^{k}$ such that for sufficiently small $\varepsilon$,

$$
\operatorname{dist}\left(x, \sigma^{l}\right)=\operatorname{dist}(x, q)=\varepsilon,
$$

but with metric on this set rescaled by a factor $1 / \varepsilon$. The latter definition makes sense if $\sigma^{k} \supset \sigma^{l}$ is replaced by $K \supset \sigma^{l}$. Then $L\left(\sigma^{l}, K\right)=L\left(\sigma^{l}\right)$ becomes the constant curvature analog of a p.l. space - a triangulated space of piecewise constant curvature $\equiv 1$. The decomposition into spherical simplices corresponds to

$$
L\left(\sigma^{l}, K\right)=\bigcup_{\sigma^{l} \subset \sigma^{k} \subset K} L\left(\sigma^{l}, \sigma^{k}\right) .
$$


Let $\operatorname{st}\left(\bar{\sigma}^{l}\right)$ be the set of all closed simplices containing $\bar{\sigma}^{l}$ as a face. Then $L\left(\bar{\sigma}^{l}\right)$ is homeomorphic to the combinatorial link [the subcomplex of $\partial\left(\operatorname{st}\left(\bar{\sigma}^{l}\right)\right)$ spanned by those vertices not belonging to $\bar{\sigma}^{l}$ ] via radial projection.

If we glue together the cones $C^{\perp}\left(\sigma^{l}, \sigma^{k}\right)$ in the obvious fashion corresponding to (1.4), we get the piecewise flat normal cone $=C^{\perp}\left(\sigma^{l}\right)$. Thus, $C^{\perp}\left(\sigma^{l}\right)=C\left(L\left(\sigma^{l}\right)\right)$.

The normalized volume of $L\left(\sigma^{l}, \sigma^{k}\right)$, is denoted by $\left(\sigma^{l}, \sigma^{k}\right)$ or simply $(l, k)$ and called the (interior) dihedral angle of $\sigma^{l} \subset \sigma^{k}$ (the normalization is such that the unit sphere in all dimensions has volume 1).

If we fix an identification of $L\left(\sigma^{l}, \sigma^{k}\right)$ with a subset of the unit $k$-sphere in $E^{k+1}$, we have the dual simplex $L\left(\sigma^{l}, \sigma^{k}\right)^{*}$ given by all unit vectors making an angle $>\pi / 2$ with every vector of $L\left(\sigma^{l}, \sigma^{k}\right)$. The normalized volume $(l, k)^{*}$ of $L\left(\sigma^{l}, \sigma^{k}\right)^{*}$ is called the exterior dihedral angle of $\sigma^{l} \subset \sigma^{k}$.

Let $C_{0,{ }_{\varepsilon}}^{\perp}\left(\sigma^{l}\right)$ denote those points in the normal cone at $q \in \sigma^{l}$ having distance $<\varepsilon$ from the vertex. It is easily seen that for $\varepsilon$ small, there are neighborhoods of $q$ in $K$ of the form

$$
U^{l} \times C_{0, \varepsilon}^{\perp}\left(\sigma^{l}\right),
$$

where $U^{l} \subset \sigma^{l}$ is flat and the metric is the product metric. This local product structure is the characteristic feature of the geometry of p.l. spaces. After splitting off a maximal flat factor from $C^{\perp}$, we arrive at the local normal structure

$$
U^{l} \times F^{r} \times \hat{C}_{0, \varepsilon}^{\perp},
$$

which is constant along the flat factor $U^{l} \times F^{r}$. It follows easily that $K$ has an intrinsic decomposition into flat open submanifolds $\Sigma_{\text {int }}^{s}$ of dimension $s$, along which the normal cone is irreducible and locally constant. This is called the intrinsic stratification of $K$ and the $\Sigma_{\text {int }}^{s}$ are called strata, compare [McC].

Recall that in general a metric cone $C$ is a complete metric space on which $R^{+}$ operates by homotheties $T_{r}^{*}\left(T_{r}\right.$ multiplies distances by $\left.r\right)$. The unique common fixed point of the action is called the vertex $p$. The cross-section $L$ (the link of $p$ ) is the set of points at distance 1 from $p$. We write $C=C(L)$.

The properties of products of p.l. spaces are conveniently stated in terms of products of general cones. Here the essential point is the existence of a space $L_{1} * L_{2}$, the metric join of $L_{1}$ and $L_{2}$ such that the metric identity

$$
C\left(L_{1} * L_{2}\right)=C\left(L_{1}\right) \times C\left(L_{2}\right)
$$

holds. The action $R^{+}$on the right hand side is the diagonal action. In terms of polar coordinates $(r, x)$ where $r \in R^{+}, x \in L$ clearly

$$
\left(r_{1}, r_{2}, x_{1}, x_{2}\right) \in C\left(L_{1}\right) \times C\left(L_{2}\right),
$$

where

$$
r_{1}^{2}+r_{2}^{2}=1 \text {. }
$$

Since $\left(0, x_{j}\right)=p_{j}$ for all $x_{j}, j=1,2$, it follows that $L_{1} * L_{2}$ is homeomorphic to $[0,1] \times L_{1} \times L_{2}$, modulo the identifications

$$
\begin{aligned}
& (0, x, y)=(0, x, y), \\
& (1, x, y)=(1, x, y)
\end{aligned}
$$

for all $x, \underline{x}, y, \underline{y}$. 
If $C\left(\tau_{1}^{k_{1}}\right), C\left(\tau_{2}^{k_{2}}\right)$ denote simplicial cones, $C\left(\tau_{1}^{k_{1}}\right) \times C\left(\tau_{2}^{k_{2}}\right)$ is again simplicial. If $l_{1}^{1} \ldots l_{k_{1}}^{1}, l_{1}^{2} \ldots l_{k_{2}}^{2}$ denote the corresponding linear functionals, then the natural extensions of all of these to $E^{k_{1}} \oplus E^{k_{2}}$ determines $C\left(\tau_{1}^{k_{1}} * \tau_{2}^{k_{2}}\right)$. It follows that the join $\tau_{1}^{l_{1}} * \tau_{2}^{l_{2}}$ of spherical simplices $\tau_{1}^{l_{1}}, \tau_{2}^{l_{2}}$ is again a spherical simplex of dimension $\left(l_{1}+l_{2}+1\right)$. Similarly the product of piecewise flat cones $C\left(L_{1}\right), C\left(L_{2}\right)$ is again piecewise flat. In this case $L_{1} * L_{2}$ is naturally a complex with spherical simplices of the 3 types of simplices: $\tau_{1}, \tau_{2}$, and $\tau_{1} * \tau_{2}$.

It follows that the corresponding Euler characteristics satisfy

$$
1-\chi\left(L_{1} * L_{2}\right)=\left(1-\chi\left(L_{1}\right)\right)\left(1-\chi\left(L_{2}\right)\right),
$$

where as usual

$$
\chi(K)=\sum_{k}(-1)^{k} \#\left(\sigma^{k}\right) .
$$

Finally, we claim the (normalized) measure $\left|\tau_{1} * \tau_{2}\right|$ of $\tau_{1} * \tau_{2}$ is the product of the normalized measures of $\tau_{1}$ and $\tau_{2}$ :

$$
\left|\tau_{1} * \tau_{2}\right|=\left|\tau_{1}\right| \cdot\left|\tau_{2}\right|
$$

Indeed, calculating the unnormalized volumes we have $\left(j_{i}=\operatorname{dim} \tau_{i}, \mathrm{k}=\mathrm{j}_{1}+\mathrm{j}_{2}+1\right)$

$$
\begin{aligned}
V\left(\tau_{1} * \tau_{2}\right)= & \frac{1}{k+1} V\left(C_{0,1}\left(L_{1} * L_{2}\right)\right) \\
= & \frac{1}{(k+1)} \int_{0}^{1} r^{j_{1}}\left(1-r^{2}\right)^{j_{2} / 2} d r \\
& \cdot V\left(C_{0,1}\left(\tau_{1}\right)\right) V\left(\left(C_{0,1}\left(\tau_{2}\right)\right)\right. \\
= & \frac{\left(j_{1}+1\right)\left(j_{2}+1\right)}{(k+1)} \int_{0}^{1} r^{j_{1}}\left(1-r^{2}\right)^{j_{2} / 2} d r \\
& \cdot V\left(\tau_{1}\right) V\left(\tau_{2}\right) .
\end{aligned}
$$

We now introduce some further notions concerning p.l. spaces. We denote by $\left|\sigma^{k}\right|$ the euclidean $k$-volume of the euclidean $k$-simplex to which ${ }^{s} \sigma^{k}$ is linearly isometric by assumption. By convention, the volume of a vertex is 1 . The mesh $\eta(\sigma)$ of a simplex is the supremum of its edge lengths. Its fatness $\Theta(\sigma)$ is the infimum of $\left|\sigma^{l}\right| \eta^{-l}$, where $\sigma^{l}$ is $\sigma$ or any of its faces. A simple exercise in linear algebra shows there are universal constants $c(k)$ such that for all dihedral angles and all face volumes of $\sigma(k=\operatorname{dim} \sigma)$

$$
\begin{gathered}
c^{-1}(k) \Theta(\sigma) \leqq \min _{\sigma^{l} \subset \sigma}\left(\sigma^{l}, \sigma\right) \leqq c(k) \Theta(\sigma), \\
\Theta(\sigma) \leqq\left|\sigma^{l}\right| \eta^{-l} \leqq c(k) \Theta(\sigma),
\end{gathered}
$$

i.e. the fatness of a simplex only becomes small, if at least one of its dihedral angles or a rescaled volume of one of its faces becomes small and vice versa. The mesh $\eta$ of a p.l. space is defined to be the supremum of all its edge lengths, and its fatness $\Theta$ is the infinum of $\left|\sigma^{k}\right| \eta^{-k}$, where $\sigma^{k}$ now runs through all simplices of the p.l. space.

A subdivision of a p.l. space $K$ is a p.l. space $K^{\prime}$ and a homeomorphism

$$
s: K^{\prime} \rightarrow K
$$


such that for every simplex $\sigma$ in $K^{\prime}$, its image $s(\sigma)$ is contained in some simplex $\underline{\sigma}$ of $K$, and $s \mid \sigma$ is linear. If we pull back the metric on $K$ to $K^{\prime}$, we obtain a triangulated piecewise flat space $\left(K^{\prime}, l^{\prime}\right)$ which is isometric to $K$. Alternatively, we sometimes identify $K^{\prime}$ with $s\left(K^{\prime}\right)$.

We now observe that any p.l. space $(K, l)$ has subdivisions $\left(K_{\eta}^{\prime}, l_{\eta}\right)$ of mesh $\eta \rightarrow 0$, whose fatness $\Theta(\eta)$ is bounded away from zero, $\Theta(\eta) \geqq \Theta_{0}>0$ (see [F, Whi] for more complicated constructions).

It suffices to consider a standard $n$-simplex $\sigma^{n}$. For each vertex $v_{j}, j=1, \ldots, n+1$, we have a rectangular solid $C_{j}$ homeomorphic to a hypercube defined by

$$
C_{j}\left\{\left(x_{1}, \ldots, x_{n}\right) \mid x_{j} \geqq x_{i} \text { for all } i\right\} \text {. }
$$

For $i \neq j$, the opposing faces of $C_{j}$ are given by the pairs of planes containing the sets

$$
\begin{aligned}
& H_{j}^{i, 0}=\left\{\left(x_{1}, \ldots, x_{n}\right) \mid x_{i}=0\right\}, \\
& H_{j}^{i, 1}=\left\{\left(x_{1}, \ldots, x_{n}\right) \mid x_{i}=x_{j}\right\} .
\end{aligned}
$$

Choose a suitable smooth interpolation $H_{j}^{i, t}$ between $H_{j}^{i, 0}, H_{j}^{i, 1}$ which sweeps out $C_{j}$, and is invariant under the permutations of $i \neq j$ (see Fig. 1.2).

Fig. 1.2

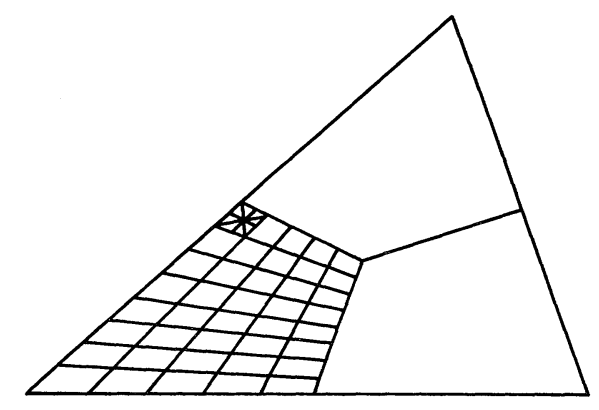

The collection of all hyperplanes $H_{j}^{i, k / N}, 0 \leqq k \leqq N, N$ large, divides $C_{j}$ into $N^{n}$ regions, each of which is approximately a parallelopiped $P$. Now form the first barycentric subdivision $P^{\prime}$ of $P$. This is the simplicial complex whose vertices are the barycenters of the faces of $P$ and whose simplices correspond to increasing sequences of faces under inclusion. The resulting sequence of complex has the properties claimed.

We turn to the concept of triangulations. A smooth triangulation of a smooth manifold $U$ is a pair $\{K, f\}$ where $K$ is a simplicial complex and $f$ is a homeomorphism $f:{ }^{s} K \rightarrow U$ such that $f \mid \bar{\sigma}$ is smooth for every closed simplex $\bar{\sigma}$ in $K$. A well known theorem says that any compact smooth manifold $U$ has a smooth triangulation with finite $K$ (see e.g. [Mu]).

Let $p$ be a point in a Riemannian manifold $M^{n}$. Assume $\exp _{p} \mid B_{1}(0) \subset M_{p}^{n}$ is a diffeomorphism and that $\|R\| \leqq 1$ on $B_{1}(p)$. Then it is easy to see that for all $\Theta_{0}>0$ there exists $\eta\left(\Theta_{0}\right)>0$ with the following property. Suppose $0, q_{1}, \ldots, q_{n}$ are the vertices of a linear simplex in $M_{p}$ with edge lengths $\tilde{l}_{i j}$. If the fatness of this simplex 
is $\geqq \Theta_{0}$ and its mesh is $\leqq \eta\left(\Theta_{0}\right)$ then the simplex with edge lengths

$$
l_{i j}=\operatorname{dist}\left(\exp _{p} q_{i}, \exp _{p} q_{j}\right)
$$

satisfies

$$
\left|l_{i j}-\tilde{l}_{i j}\right| \leqq C \eta^{2} .
$$

Moreover, the $l_{i j}$ are the edge lengths of a simplex of fatness $\geqq \Theta_{0} / 2$ (compare the discussion below).

If $K \stackrel{f}{\rightarrow} M^{n}$ is a smooth triangulation of $M^{n}$ as above and $K^{\prime} \stackrel{s}{\rightarrow} K$ is any sufficiently fine fat subdivision, for example the one constructed above, then it is easy to see that the conditions of the preceding paragraph will be satisfied for all simplices with

$$
l_{i j}=\operatorname{dist}\left(f \circ s\left(\sigma_{i}^{0}\right), f \circ s\left(\sigma_{j}^{0}\right)\right),
$$

where $\sigma_{i}^{0}, \sigma_{j}^{0}$ span a 1 -simplex of $K^{\prime}$. For any such triangulation one can construct an associated p.l. space

$$
M_{\eta}=\left(K^{\prime},\left\{l_{i j}\right\}\right) .
$$

The spaces $M_{\eta}$ are the piecewise flat approximations to smooth Riemannian spaces which are the central objects of study in this paper.

If $M$ is a manifold with boundary we modify our construction and use lengths of boundary geodesics whenever the vertices of a 1-simplex are points of the boundary. However, one can show that the results in Sect. 8 on boundary Lipschitz-Killing curvatures remain valid, if the approximating p.l. spaces are constructed by using lengths of geodesics in the manifold itself only. More generally, if $M^{n}$ is isometrically embedded in a manifold $\bar{M}^{m}(n \leqq m)$, then the lengths of ambient geodesics in $\bar{M}^{m}$ may be used. For the particular case where $\bar{M}^{m}$ is a euclidean space $E^{m}$, the resulting p.l. space is called a secant approximation to $M^{n}$.

The proof of our main results remains essentially unchanged with these alternative choices of lengths.

\section{Linear Algebra}

Here we derive some basic formulas which are important for later sections. The main results are (2.20) and (2.28).

Let $V^{n}$ be a vector space and let $\Lambda^{i}\left(V^{n}\right)$ denote the $i^{\text {th }}$ exterior power of $V^{n}$. Let $0 \neq \omega \in \Lambda^{n}\left(V^{n}\right)$ be a volume element. Then there is an isomorphism (Lefschetz duality)

$$
\beta_{\omega}: \Lambda^{i}\left(V^{n}\right) \rightarrow\left(\Lambda^{n-i}\left(V^{n}\right)\right)^{*}
$$

defined by

$$
\beta_{\omega}\left(\phi_{i}\right)\left(\phi_{n-i}\right) \omega=\phi_{i} \wedge \phi_{n-i}
$$

Clearly, for $k \neq 0$,

$$
\beta_{k \omega}=\frac{1}{k} \beta_{\omega} .
$$


There is also a natural isomorphism

$$
\gamma:\left(\Lambda^{n-i}\left(V^{n}\right)\right)^{*} \rightarrow \Lambda^{n-i}\left(V^{n^{*}}\right) .
$$

Since

$$
\gamma: V^{n^{*}} \rightarrow \Lambda^{1}\left(V^{n^{*}}\right)
$$

then

$$
\beta_{\omega}^{-1}: V^{n^{*}} \rightarrow \Lambda^{n-1}\left(V^{n}\right)
$$

and

$$
\left(\beta_{\omega}^{-1}\right)^{n-i}: \Lambda^{n-i}\left(V^{n^{*}}\right) \rightarrow \Lambda^{n-i}\left(\Lambda^{n-1}\left(V^{n}\right)\right) .
$$

Thus, setting

$$
\alpha_{\omega}=\left(\beta_{\omega}^{-1}\right)^{n-i} \gamma \beta_{\omega}
$$

we have the isomorphism

$$
\alpha_{\omega}: \Lambda^{i}\left(V^{n}\right) \rightarrow \Lambda^{n-i}\left(\Lambda^{n-1}\left(V^{n}\right)\right)
$$

and

$$
\alpha_{k \omega}=k^{n-i-1} \alpha_{\omega} .
$$

If $x_{1}, \ldots, x_{m}$ is a basis for $X^{m}$, let $x_{1}^{*}, \ldots, x_{n}^{*}$ be the dual basis. Let $v_{1}, \ldots, v_{n}$ be an ordered basis for $V^{n}$ and let $\omega=v_{1} \wedge \ldots \wedge v_{n}$. Then

$$
\gamma \beta_{\omega}\left(v_{j_{1}} \wedge \ldots \wedge v_{j_{i}}\right)=(-1)^{\tau(j)}\left(v_{1}^{*} \wedge \ldots \wedge \hat{v}_{j_{1}}^{*} \wedge \ldots \wedge \hat{v}_{j_{i}}^{*} \wedge \ldots \wedge v_{n}^{*}\right),
$$

where $(j)=\left(j_{1}, \ldots, j_{i}\right)$ and

$$
(-1)^{\tau(j)} v_{j_{1}} \wedge \ldots \wedge v_{j_{2}} \wedge v_{1} \wedge \ldots \wedge \hat{v}_{j_{1}} \wedge \ldots \wedge \hat{v}_{j_{2}} \wedge \ldots \wedge v_{n}=\omega
$$

Set

$$
w_{l}=(-1)^{l} v_{1} \wedge \ldots \wedge \hat{v}_{l} \wedge \ldots \wedge v_{n}
$$

Then

$$
\beta_{\omega}^{-1}\left(v_{l}^{*}\right)=(-1)^{n} w_{l}
$$

and

$$
\begin{aligned}
& \left(\beta_{\omega}^{-1}\right)^{n-i}\left(v_{1}^{*} \wedge \ldots \wedge \hat{v}_{j_{1}}^{*} \wedge \ldots \wedge \hat{v}_{j_{l}}^{*} \wedge \ldots \wedge v_{n}^{*}\right) \\
& \quad=(-1)^{n(n-i)} w_{1} \wedge \ldots \wedge \hat{w}_{j_{1}} \wedge \ldots \wedge \hat{w}_{j_{i}} \wedge \ldots \wedge w_{n}
\end{aligned}
$$

Thus

$$
\alpha_{\omega}\left(v_{j_{1}} \wedge \ldots \wedge v_{j_{i}}\right)=(-1)^{n(n-i)+\tau(j)} w_{1} \wedge \ldots \wedge \hat{w}_{j_{1}} \wedge \ldots \wedge \hat{w}_{j_{i}} \wedge \ldots \wedge w_{n}
$$

Now choose an inner product $\langle$,$\rangle on V^{n}$ and let $\Omega$ be the associated volume element. Clearly

$$
\frac{1}{\left\|v_{1} \wedge \ldots \wedge v_{n}\right\|} \omega=\Omega
$$


so that by $(2.10)$,

$$
\left\|v_{1} \wedge \ldots \wedge v_{n}\right\|^{n-i-1} \alpha_{\Omega}=\alpha_{\omega} .
$$

Moreover, $\alpha_{\Omega}$ is an isometry with respect to the standard inner products. Recall that the inner product on $\Lambda^{l}\left(V^{n}\right)$ is defined by

$$
\left\langle v_{1} \wedge \ldots \wedge v_{l}, u_{1} \wedge \ldots \wedge u_{l}\right\rangle=\operatorname{det}\left(v_{i}, u_{j}\right) .
$$

In particular, taking $i=n-2$ in (2.9),

$$
\begin{aligned}
& \left\|v_{1} \wedge \ldots \wedge v_{n}\right\| \cdot\left\|v_{1} \wedge \ldots \wedge \hat{v}_{i} \wedge \ldots \wedge \hat{v}_{j} \wedge \ldots \wedge v_{n}\right\| \\
& \quad=\left\|w_{i} \wedge w_{j}\right\| \\
& \quad=\left\|w_{i}\right\| \cdot\left\|w_{j}\right\| \sin \Theta_{i j}
\end{aligned}
$$

where $\Theta_{i j}$, the angle between the hyperplanes determined by $w_{i}, w_{j}$, is defined by

$$
\pi-\Theta_{i j}=\cos ^{-1}\left(\frac{\left\langle w_{i}, w_{j}\right\rangle}{\left\|w_{i}\right\| \cdot\left\|w_{j}\right\|}\right) .
$$

Now consider the $n$-simplex $\sigma^{n}$ which is determined by the $\left\{v_{i}\right\}$. By definition the vertices of $\sigma^{n}$ are the 0 -vector (origin), together with $v_{1}, \ldots, v_{n}$. If we set $v_{0}=0$, the $i$-faces $\sigma_{(j)}^{i}$ of $\sigma^{n}$ are the sets of convex combinations

$$
\sum_{k=1}^{i} y_{k} v_{j_{k}}
$$

where $0 \leqq j_{1}<j_{2}<\ldots<j_{i} \leqq n, \quad(j)=\left(j_{1}, \ldots, j_{i}\right), \quad y_{k}>0, \quad \sum y_{k}=1 . \quad$ Denote the $i$-dimensional volume of $\sigma^{i}$ by $\left|\sigma^{i}\right|$. Let the $(n-1)$-faces of $\sigma^{n}$ be oriented by the inward normal. Those that contain the origin correspond to $(j)=\left(0=j_{1}, \ldots, \hat{l} \ldots\right)$. Denote them by $\sigma_{l}^{n-1}, l=1, \ldots, n$. They determine elements $\frac{1}{(n-1) !} w_{l} \in \Lambda^{n-1}\left(V^{n}\right)$ in the following sense. The subspace determined by $v_{1}, \ldots, \hat{v}_{l}, \ldots, v_{n}$ is the $(n-1)$ dimensional subspace containing $\sigma_{l}^{n-1}$, the orientations are compatible, and $\frac{1}{(n-1) !} \| w_{l}||=\left|\sigma^{n-1}\right|$. In the same sense, the remaining face $\sigma_{0}^{n-1}$ corresponds to $w_{0}$, which for any $l=1, \ldots, n$, can be written as

$$
w_{0}=(-1)^{l+1}\left(v_{1}-v_{l}\right) \wedge \ldots \wedge\left(v_{l-1}-v_{l}\right) \wedge\left(v_{l+1}-v_{l}\right) \wedge \ldots \wedge\left(v_{n}-v_{l}\right) .
$$

It follows from (2.13), (2.23) that

$$
\sum_{0}^{n} w_{l}=0
$$

Similarly, $\sigma^{n}$ itself corresponds to $\frac{1}{n !} v_{1} \wedge \ldots \wedge v_{n}$ and

$$
\left|\sigma^{n}\right|=\frac{1}{n !}\left\|v_{1} \wedge \ldots \wedge v_{n}\right\|
$$


We now explain a remarkable variational formula of Regge, [R], (the generalization of which will play a crucial role in the proof of our main result. Let $\Theta_{i j}$ be as in (2.21), $(0 \leqq i<j \leqq n)$ and consider the quantity

$$
\sum_{\sigma_{i, j}^{n-2}<\sigma^{n}} \Theta_{i j}\left|\sigma_{i j}^{n-2}\right|
$$

where $\sigma_{i, j}^{n-2}=\sigma_{i}^{n-1} \cap \sigma_{j}^{n-1}$. Let the $\left\{v_{i}\right\}$ depend on a parameter $t$. By Leibnitz' rule,

$$
\left(\sum \Theta_{i j}\left|\sigma_{i j}^{n-2}\right|\right)^{\prime}=\sum \Theta_{i j}^{\prime}\left|\sigma_{i j}^{n-2}\right|+\sum \Theta_{i j}\left|\sigma_{i j}^{n-2}\right|^{\prime} .
$$

However, according to Regge, the first term in (2.27) vanishes identically for all variations. So in fact

$$
\left(\sum \Theta_{i j}\left|\sigma_{i j}^{n-2}\right|\right)^{\prime}=\sum \Theta_{i j}\left|\sigma_{i j}^{n-2}\right|^{\prime} .
$$

To see (2.28), take the inner product of (2.24) with $\frac{-w_{j}}{\left\|w_{j}\right\|}$ to obtain

$$
0=\frac{-1}{\left\|w_{j}\right\|} \sum_{i=0}^{n}\left\langle w_{i}, w_{j}\right\rangle=\sum_{i=0}^{n}\left\|w_{i}\right\| \cos \Theta_{i j}
$$

Differentiate (2.29) with respect to $t$, multiply by $\left\|w_{j}\right\|$ and sum over $j$ to get

$$
0=\sum_{j} \sum_{i}\left\|w_{j}\right\|\left(\left\|w_{i}\right\|^{\prime} \cos \Theta_{i j}-\left\|w_{i}\right\| \sin \Theta_{i j} \Theta_{i j}^{\prime}\right)
$$

If we reverse the order of summation in (2.30) and use (2.29) with $i$ and $j$ interchanged, we see that the first term of (2.30) vanishes. Then using (2.20) and the relation

$$
\left|\sigma_{i j}^{n-2}\right|=\frac{1}{(n-1) !}\left\|v_{1} \wedge \ldots \wedge \hat{v}_{i} \wedge \ldots \wedge \hat{v}_{j} \wedge \ldots \wedge v_{n}\right\|,
$$

the second term of $(2.30)$ is seen to equal $\frac{1}{(\mathrm{n}-2) !}\left\|v_{1} \wedge \ldots \wedge v_{n}\right\|$ times the first term of (2.27). This gives (2.28).

\section{Lipschitz-Killing Curvatures for Piecewise Flat Spaces}

In Sect. 0 it was pointed out that the analogs of Lipschitz-Killing curvatures for piecewise flat spaces must exhibit the same scaling properties and behavior under metric products as in the smooth case. In particular, the piecewise flat analog of $R^{j}$ must scale like $c^{n-j}$, when distances are multiplied by $c$. Now as in Sect. 1, if $p \in \sigma^{i}$ is a point in a piecewise flat space $X^{n}$, then $p \in U^{i} \times C_{\varepsilon}^{\perp}\left(\sigma^{i}\right)$, where $U^{i} \subset \sigma^{i}$ is flat, $C_{\varepsilon}^{\perp}\left(\sigma^{i}\right)$ is the normal cone, and the metric is a product. Thus, it follows that any locally computable invariant which scales like $c^{i}$, is of the form

$$
\sum_{\sigma^{i}} \psi\left(C^{\perp}\left(\sigma^{i}\right)\right) \cdot\left|\sigma^{i}\right|
$$


where $\psi\left(C^{\perp}\left(\sigma^{i}\right)\right)$ is some invariant of $C^{\perp}\left(\sigma^{i}\right)$. Suppose that we normalize $R^{j}$ in the smooth case by setting

$$
\begin{aligned}
R^{j}\left(M^{j}\right) & =P_{\chi}\left(M^{j}\right), \\
R^{j}\left(M^{j} \times E^{n-j}\right) & =P_{\chi}\left(M^{j}\right) \times \omega_{E^{n-\jmath}} .
\end{aligned}
$$

Here $P_{\chi}$ is the Chern-Gauss-Bonnet form, and $\omega_{E^{n-j}}$ is the volume form of $E^{n-j}$. Then it follows that for $R^{j}$, we have $i=n-j$. Moreover, $\psi$ must be the analog $P_{\chi}\left(C^{\perp}\left(\sigma^{n-j}\right)\right)$ of $P$ for piecewise flat spaces of $\operatorname{dim} j$, evaluated at the vertex of the $j$-dimensional piecewise flat cone $C^{\perp}\left(\sigma^{n-j}\right)$.

In seeking the analog of $P_{\chi}$, we begin with the case of dimension 2. Then $L\left(\sigma^{0}\right)=S_{\beta / 2 \pi}^{1}$, a circle of radius $\beta / 2 \pi$. If $\mathscr{V}$ is a smooth riemannian 2-disc a neighborhood of whose boundary is isometric to a neighborhood of the boundary of $C_{0, \varepsilon}\left(S_{\beta / 2 \pi}^{1}\right)$, by the Gauss-Bonnet formula,

$$
1=\frac{1}{2 \pi} \int_{\mathscr{V}} K+\beta / 2 \pi
$$

Thus, in the piecewise flat case we should have

$$
P_{\chi}\left(C^{\perp}\left(\sigma^{0}\right)\right)=1-\frac{\beta}{2 \pi} .
$$

There is another way of arriving at this conclusion which generalizes immediately to higher dimensions. It is easy to see that for any $i$-simplex we have

$$
\sum(0, i)^{*}=1,
$$

where the sum is over all $i$-dimensional exterior angles, $(0, i)^{*}$, of $\sigma^{i}$. Then for any piecewise flat space, we have the obvious formula

$$
\chi\left(M^{n}\right)=\sum_{\sigma^{i}}(-1)^{i} \sum_{\sigma^{0} \subset \sigma^{i}}(0, i)^{*}=\sum_{\sigma^{0}} \sum_{\sigma^{0} \subset \sigma^{i}}(-1)^{i}(0, i)^{*},
$$

where $(0, i)^{*}$ is the exterior angle of $\sigma^{i}$ at $\sigma^{0} C \sigma^{i}$. So we will set

$$
P_{\chi}\left(C^{\perp}\left(\sigma^{0}\right)\right)=\sum_{\sigma^{0} \subset \sigma^{i}}(-1)^{i}(0, i)^{*},
$$

and more generally

$$
P_{\chi}\left(C^{\perp}\left(\sigma^{n-j}\right)\right)=\sum_{\sigma^{n-j}<\sigma^{2}}(-1)^{i-(n-j)}(n-j, i)^{*} .
$$

This formula was emphasized by Brin $[\mathrm{Br}]$ and Banchoff $[\mathrm{B}] .{ }^{4}$ Moreover, the analog of $R^{j}$ to which it leads via (3.1)-(3.3), was shown by Wintgen [Wi] to arise in the generalization of Chern's kinematic formula to piecewise flat spaces (see [CMS2]).

It is not immediately obvious that the linear expression (3.8) is intrinsic, nor in particular that it vanishes for cones isometric to $E^{n}$. More generally, the precise behavior of the expression for piecewise flat cones which are close to $E^{n}$ is difficult to discern. For this reason, it is desirable to express (3.8) in terms of interior

4 It appears to go back much further 
dihedral angles (compare [Mc]). Such an expression can be obtained from the formula giving the relation between the values of a solid angle and its dual.

Let the convex combinations of $v_{1}, \ldots, v_{i}$ span a cone $C$ in $E^{i}$. Let $v_{j}^{*}$ denote the outward normal to the hyperplane $H_{j}$ spanned by $v_{1}, \ldots, \hat{v}_{j}, \ldots, v_{i}$, which points to the side of $H_{j}$ opposite to that of $v_{j}$. The intersection of $C$ with the unit sphere $S_{1}^{i-1} \subset E^{i}$ determines a solid angle with value $(0, i)$. The dual cone spanned by the $\left\{v_{j}^{*}\right\}$ determines the dual angle

$$
(0, i)^{*} \text {. }
$$

Moreover, $E^{i}$ is divided in $2^{i}$ closed cones $C$ which intersect only along their boundaries. These are spanned by all subsets

$$
v_{j_{1}}, \ldots, v_{j_{l}}, \quad v_{k_{1}}^{*}, \ldots, v_{k_{i-l}}^{*},
$$

where

$$
\left\{k_{1}, \ldots, k_{i-l}\right\}=\left\{j_{1}, \ldots, j_{l}\right\}^{*},
$$

is the set of indices complimentary to $\left\{j_{1}, \ldots, j_{l}\right\}$. Then apart from the cases corresponding to (3.9), (3.10), each such $C$ is the metric product of the cones determined by $v_{j_{1}}, \ldots, v_{j_{l}}$ and $v_{k_{1}}^{*}, \ldots, v_{k_{2}-l}^{*}$. If $N, N^{\perp}$ denote the cross-sections of these cones, then the cross section of the product cone is $N * N^{\perp}$, the metric join of $N, N^{\perp}$, whose (normalized) volume, by (1.18), satisfies

$$
\left|N * N^{\perp}\right|=(0, l)(l, i)^{*} \text {. }
$$

Thus, by adding the volumes of all such regions we arrive at the well known formula

$$
(0, i)+(0, i-1)(i-1, i)^{*}+(0, i-2)(i-2, i)^{*}+\ldots+(0, i)^{*}=1
$$

(the sum is over all cones as above). In the same way, we have the more genral relation

$$
(l, i)+(l, i-1)(i-1, i)^{*}+\ldots+(l, i)^{*}=1 .
$$

If we substitute (3.15) in (3.14) and iterate, we arrive at the relation

$$
(0, i)^{*}=1-\sum(-1)^{l}\left(0, i_{1}\right)\left(i_{1}, i_{2}\right) \ldots\left(i_{l-1}, i_{l}\right)
$$

where the sum is over all

$$
v_{1} \ldots v_{i_{1}} \subset v_{1} \ldots v_{i_{1}} \ldots v_{i_{2}} \subset \ldots \subset v_{1} \ldots v_{i_{1}} \ldots v_{i_{l}},
$$

$0<i_{1}<i_{2}<\ldots<i_{l}$. Thus, the expression in (3.8) becomes

$$
P_{\chi}\left(C^{\perp}\left(\sigma^{0}\right)\right)=\phi(0)+\sum(-1)^{l+i_{l}}\left(0, i_{1}\right)\left(i_{1}, i_{2}\right) \ldots\left(i_{l-1}, i_{l}\right) \phi\left(i_{l}\right)
$$

where

$$
\phi(j)=1-\chi\left(L\left(\sigma^{j}\right)\right)
$$

We can also rewrite (3.18) as

$$
\sum_{i_{l}<n}(-1)^{l}(0, i) \ldots\left(i_{l-1}, i_{l}\right)\left((-1)^{i_{l}} \phi\left(i_{l}\right)+(-1)^{n-1} \sum_{\sigma^{i_{l}<\sigma^{n}}}\left(i_{l}, \mathrm{n}\right)\right) .
$$


Suppose in particular that $\sigma^{0}$ is an interior point of a manifold $M^{n}$ which is flat near $\sigma^{0}$, then

$$
\sum_{\sigma^{l_{l} \subset \sigma^{n}}}\left(i_{l}, n\right)=1
$$

and

$$
\begin{aligned}
(-1)^{i_{l}} \phi\left(\sigma^{i_{l}}\right)+(-1)^{n+1} \sum_{\sigma_{l_{l} \subset \sigma^{n}}}\left(i_{l}, n\right) \\
\quad=(-1)^{i_{l}}\left(1-\chi\left(S^{n-i_{l}-1}\right)+(-1)^{n-i_{l}+1}\right) \\
\quad=0 .
\end{aligned}
$$

Similarly, (3.20) vanishes if $\sigma^{0}$ is a boundary point near which $M^{n}$ is isometric to a half space.

In view of (3.18), the definition of $R^{j}$ given in (3.1) can also be written as

$$
\begin{aligned}
R^{j}= & \sum_{\sigma^{n-j}}\left\{\phi(n-j)+\sum(-1)^{l}\left(n-j, n-j+i_{1}\right) \ldots\left(n-j+i_{l-1}, n-j+i_{l}\right)\right. \\
& \left.\cdot \phi\left(n-j+i_{l}\right)\right\}\left|\sigma^{n-j}\right| .
\end{aligned}
$$

We can use (3.23) to see that $R^{j}$ has the same behavior under metric products as in the smooth case. Let $X^{n_{1}}, X^{n_{2}}$ be piecewise flat spaces. As explained in Sect. 2, $X^{n_{1}} \times X^{n_{2}}$ has a piecewise flat stratification such that

$$
\Sigma^{i}\left(X^{n_{1}} \times X^{n_{2}}\right)=\bigcup_{k} \Sigma^{k}\left(X^{n_{1}}\right) \times \Sigma^{i-k}\left(X^{n_{2}}\right) .
$$

If $\sigma_{1}^{k} \in \Sigma^{k}\left(X^{n_{1}}\right), \sigma_{2}^{i-k} \in \Sigma^{i-k}\left(X^{n_{2}}\right)$, then

$$
C^{\perp}\left(\sigma_{k}^{k} \times \sigma_{2}^{i-k}\right) \cong C^{\perp}\left(\sigma_{1}^{k}\right) \times C^{\perp}\left(\sigma_{2}^{i-k}\right)
$$

where the product is the metric product. As in (1.7),

$$
L\left(\sigma_{1}^{k} \times \sigma_{2}^{i-k}\right)=L\left(\sigma_{1}^{k}\right) * L\left(\sigma_{2}^{i-k}\right) .
$$

Thus, $L\left(\sigma_{1}^{k} \times \sigma_{2}^{i-k}\right)$ inherits a triangulation the simplices of which are joins $\tau_{1}^{\alpha_{1}} * \tau_{2}^{\alpha_{2}}$ of spherical simplices $\tau_{1}^{\alpha_{1}}, \tau_{2}^{\alpha_{2}}$ of $L\left(\sigma_{1}^{k}\right)$ and $L\left(\sigma_{2}^{i-k}\right)$. By (1.13), the normalized volume of $\tau_{1}^{\alpha_{1}} * \tau_{2}^{\alpha_{2}}$ is given by the product of the normalized volumes of $\tau_{1}^{\alpha_{1}}, \tau_{2}^{\alpha_{2}}$. Moreover, as in (1.12)

$$
\chi(A * B)=-\chi(A) \chi(B)+\chi(A)+\chi(B),
$$

from which it follows that

$$
\phi\left(\sigma_{1}^{k} \times \sigma_{2}^{i-k}\right)=\phi\left(\sigma_{1}^{k}\right) \times \phi\left(\sigma_{2}^{i-k}\right) .
$$

This is the first step in proving

$$
P_{\chi}\left(C^{\perp}\left(\sigma_{1}^{k} \times \sigma_{2}^{j-k}\right)\right)=P_{\chi}\left(C^{\perp}\left(\sigma_{1}^{k}\right)\right) P_{\chi}\left(C^{\perp}\left(\sigma_{2}^{j-k}\right)\right) .
$$

To proceed with the proof, we look at the contribution [see (3.18)] to the left hand side of (3.29)

$$
\begin{aligned}
& (-1)^{l}\left(\sigma_{1}^{k} \times \sigma_{2}^{j-k}, \sigma_{1}^{k_{1}} \times \sigma_{2}^{j+i_{1}-k_{1}}\right) \ldots \\
& \quad \ldots\left(\sigma_{1}^{k_{l}-1} \times \sigma_{2}^{j+i_{l-1}-k_{l-1}}, \sigma_{1}^{k_{l}} \times \sigma_{2}^{j+i_{l}-k_{l}}\right) \phi\left(\sigma_{1}^{k_{l}} \times \sigma_{2}^{j+i_{l}-k_{l}}\right),
\end{aligned}
$$


given by a sequence

$$
\sigma_{1}^{k} \times \sigma_{2}^{j-k} \subset \sigma_{1}^{k_{1}} \times \sigma_{2}^{j+i_{1}-k_{1}} \subset \ldots \subset \sigma_{1}^{k_{l}} \times \sigma_{2}^{j+i_{l}-k_{l}}
$$

of length $l$, which is strictly increasing, i.e.

$$
0<i_{1}<i_{2}<\ldots<i_{l} .
$$

The projection of the sequence (3.31) onto each of its factors defines two strictly increasing sequences

$$
\begin{gathered}
\sigma_{1}^{k} \subset \sigma_{1}^{p_{1}} \subset \ldots \subset \sigma_{1}^{p_{l_{1}}}, \\
\sigma_{2}^{j-k} \subset \sigma_{2}^{q_{1}} \subset \ldots \subset \sigma_{2}^{q_{l_{2}}} .
\end{gathered}
$$

Now fix the sequences in (3.32) and consider the family of all sequences of the form (3.31) which project onto these two fixed sequences. Apart from the factor $(-1)^{l}$, all of these give the same contribution as the term in (3.30), since by (3.26), and (1.14)

$$
\left(\sigma_{1}^{j_{1}} \times \sigma_{2}^{j_{2}}, \sigma_{1}^{j_{1}^{\prime}} \times \sigma_{2}^{j_{2}^{\prime}}\right)=\left(\sigma_{1}^{j_{1}}, \sigma_{1}^{j_{1}^{\prime}}\right)\left(\sigma_{2}^{j_{2}}, \sigma_{2}^{j_{2}^{\prime}}\right) .
$$

Now an easy argument by induction shows that $\sum(-1)^{l}=(-1)^{l_{1}+l_{2}}$, where the summation is over the members of this family. Indeed, subdivide this family according to the form of $\sigma_{1}^{k_{l-1}} \times \sigma_{2}^{i+i_{l-1}-k_{l-1}}$. There are three possibilities

$$
\begin{gathered}
\sigma_{1}^{p_{l_{1}-1}} \times \sigma_{2}^{q_{l_{2}-1}}, \\
\sigma_{1}^{p_{l_{1}}} \times \sigma_{2}^{q_{l_{2}-1}}, \\
\sigma_{1}^{p_{l_{1}-1}} \times \sigma_{2}^{q_{l_{2}}} .
\end{gathered}
$$

By induction assumption, the contributions to $\sum(-1)^{l}=\sum(-1)(-1)^{l-1}$ from the cases $(1),(2)$, and $(3)$ are $(-1)(-1)^{l_{1}+l_{2}-2},(-1)(-1)^{l_{1}+l_{2}-1}$ and $(-1)(-1)^{l_{1}+l_{2}-1}$, respectively, adding up to $(-1)^{l_{1}+l_{2}}$. Therefore the sum of (3.30) over this family corresponds to exactly one contribution to the right hand side of (3.29), proving equality. Hence,

$$
R^{j}\left(X^{n_{1}} \times X^{n_{2}}\right)=\sum_{k=0}^{j} R^{k}\left(X^{n_{1}}\right) \cdot R^{j-k}\left(X^{n_{2}}\right),
$$

where the equality is in the sense of measures. This is the same relation as holds in the smooth case.

Corollary 3.1. Let $X^{n}=X^{n-m} \times E^{m}$. Then $R^{k}\left(X^{n}\right) \equiv 0$ for $k>n-m$.

We can also give an expression for (3.18), solely in terms of even dimensional interior angles,

$$
P_{\chi}\left(C^{\perp}\left(\sigma^{0}\right)\right)=\chi^{\perp}(0)+\sum(-1)^{l}\left(0,2 i_{1}\right)\left(2 i_{1}, 2 i_{2}\right) \ldots\left(2 i_{l-1}, 2 i_{l}\right) \chi^{\perp}\left(2 i_{l}\right)
$$

where

$$
\chi^{\perp}(j)= \begin{cases}1-\frac{1}{2} \chi\left(L\left(\sigma^{j}\right)\right) & j<n, \\ 1 & j=n .\end{cases}
$$


Let $k=[n / 2]$. If $i_{l}<k$, we set

$$
\Delta\left(2 i_{l}\right)=\chi^{\perp}\left(2 i_{l}\right)-\sum_{\sigma^{2 i_{l} \subset} \sigma^{2 k}}\left(2 i_{l}, 2 k\right) \chi^{\perp}(2 k) .
$$

Then (3.35) can also be written as

$$
\sum_{i_{l}<k}(-1)^{l}\left(0,2 i_{1}\right) \ldots\left(2 i_{l-1}, 2 i_{l}\right) \Delta\left(2 i_{l}\right) .
$$

The expressions (3.35), (3.38) were originally derived in [C] by heat equation methods. We note that these methods formally imply (3.29) (see [C, p. 111]). Note that (3.35) reduces to (3.5), for $n=2$. In the same way, we have the alternative formulas

$$
\begin{aligned}
R^{j}= & \sum_{0<l, 2 i_{l}<j}(-1)^{l}\left(n-j, n-j+2 i_{1}\right) \ldots\left(n-j+2 i_{l-1}, n-j+2 i_{l}\right) \\
& \cdot \Delta\left(n-j+2 i_{l}\right)\left|\sigma^{n-j}\right|
\end{aligned}
$$

and

$$
\begin{aligned}
R^{j}= & \sum_{\sigma^{n-j}}\left\{\chi^{\perp}\left(\sigma^{n-j}\right)+\sum_{0<l, 2 i_{l} \leqq j}(-1)^{l}\left(n-j, n-j+2 i_{1}\right) \ldots\right. \\
& \left.\ldots\left(n-j+2 i_{l-1}, n-j+2 i_{l}\right) \chi^{\perp}\left(n-j+2 i_{l}\right)\right\}\left|\sigma^{n-j}\right| .
\end{aligned}
$$

In particular, suppose the p.l. space is also a topological manifold with boundary. Then for $n-k$ odd, $\chi^{\perp}(k)$ is zero if $\sigma^{k}$ is not contained in the boundary and $\frac{1}{2}$ otherwise. Therefore, for $j$ odd, the only nonzero contributions to $R^{j}$ come from simplexes $\sigma^{n-j}$ contained in the boundary. These simplices contribute half the corresponding $(j-1)^{\text {st }}$ Lipschitz-Killing curvature of the boundary. The corresponding fact in the smooth case is well known.

In order to prove that (3.23) is equivalent to (3.39) we note first that it suffices to consider the case of a cone whose cross section is a single spherical simplex, $\tau^{n-1}$. To see this, consider two piece-wise flat cones $A, B$ which intersect in a piecewise flat cone of lower dimension. Then if $\psi$ denotes either the expression in (3.18), (3.35), the relation $\chi(X \cup Y)=\chi(X)+\chi(Y)-\chi(X \cap Y)$ gives

$$
\psi(A \cup B)=\psi(A)+\psi(B)-\psi(A \cap B) .
$$

Then the equivalence for $n$-dimensional cones follows from the equivalence for $(n-1)$-dimensional cones, the equivalence for cones with cross section $\tau^{n-1} \subset S_{1}^{n-1}$, and induction.

To obtain the equivalence for cones with cross-section $\tau^{n-1}$, we will show that (3.18), (3.35) have the same derivative when the edge lengths of $\tau^{n-1}$ are varied. The derivative formulas are also important for later sections. It then suffices to observe that both expressions vanish for the limiting case in which $\tau^{n-1}$ is a hemisphere.

Let $\tau_{t}^{n-1}$ be a 1 -parameter family of spherical $(n-1)$-simplices, having normalized volumes $(0, n)_{t}$. Then according to a classical formula of Schläfli, [Sc1, Sc2, K], the derivative, $(0, n)^{\prime}$, is given by

$$
(0, n)^{\prime}=\sum_{\tau^{n-3} \subset \tau^{n-1}}(0, n-2)(n-2, n)^{\prime} .
$$


Now differentiate a 1-parameter family of expressions as in (3.35) by employing Leibnitz' rule. The result is a sum of terms, each of which involves a product of angles, precisely one of the factors of which, say $\left(2 i_{j-1}, 2 i_{j}\right)$ is primed. Divide the collection of all such terms into two disjoint subsets, $S_{1}$ and $S_{2}$, according as to whether $i_{j} \neq i_{j-1}+1$ or $i_{j}=i_{j-1}+1$, respectively. Using (3.42), for each term in $S_{1}$ we can write

$$
\left(2 i_{j-1}, 2 i_{j}\right)^{\prime}=\left(2 i_{j-1}, 2 i_{j}-2\right)\left(2 i_{j}-2,2 i_{j}\right)^{\prime},
$$

with the appropriate sum understood. It is then apparent that each term in $S_{1}$ is cancelled by a unique term of $S_{2}$. Moreover, all terms of $S_{2}$ except those starting with $(0,2)^{\prime}$, are cancelled in this fashion. Thus, the derivative of the expression in (3.35) is given by

$$
\sum_{i_{l}<k}(-1)^{l}(0,2)^{\prime}\left(2,2 i_{2}\right) \ldots\left(2 i_{l-1}, 2 i_{l}\right) \Delta\left(2 i_{l}\right) .
$$

Note that we can rewrite (3.44) as

$$
P_{\chi}\left(C^{\perp}\left(\sigma^{0}\right)\right)^{\prime}=-\sum(0,2)^{\prime} P_{\chi}\left(C^{\perp}\left(\sigma^{2}\right)\right) .
$$

If we apply the same considerations to (3.18), we also find terms involving $i_{j}=i_{j+1}$, for which we have

$$
\begin{aligned}
& \left(i_{j-1}, i_{j-1}+1\right)_{t} \equiv \frac{1}{2}, \\
& \left(i_{j-1}, i_{j-1}+1\right)^{\prime}=0 .
\end{aligned}
$$

Thus, the $\left(i_{j-1}, i_{j-1}+1\right)^{\prime}$ play no role and an argument analogous to that above gives

$$
\sum(-1)^{l+i_{l}}(0,2)^{\prime}\left(2, i_{2}\right) \ldots\left(i_{l-1}, i_{l}\right) \phi\left(i_{l}\right)
$$

Again the unprimed part of the expression represents the negative of weight assigned by (3.18) corresponding to $P_{\chi}$ for the normal cone to $C\left(\tau^{1}\right)$. By induction we can assume that (3.18), (3.35) agree for cones of dimension $(n-2)$ and it follows that the expressions in (3.18), (3.35) coincide.

Note that if the cone corresponding to $t=0$ is actually isometric to $E^{n}$, it follows that all normal cones to the $C\left(\tau^{1}\right)$ are isometric to $E^{n-2}$. In this case, we have for all $i_{l}$,

$$
P_{\chi}\left(C^{\perp}\left(\sigma^{i_{l}}\right)\right)=0
$$

Thus, the first derivative (3.47) of (3.35) vanishes at a flat configuration. More generally, we have

Proposition 3.2. Let $C_{t}^{n}$ be a 1-parameter family of piecewise flat cones, such that $C_{0}^{n}$ is isometric to $E^{n}$ or to a half space. Then at $t=0$, the first $k-1$ derivatives of the expression in (3.35) vanish, $(k=[n / 2])$. Moreover, the $k^{\text {th }}$ derivative is given by

$$
(-1)^{k} \sum(0,2)^{\prime}(2,4)^{\prime} \ldots(2 k-2,2 k)^{\prime} P_{\chi}\left(C^{\perp}\left(\sigma^{2 k}\right)\right) .
$$

Proof. An argument like that above shows that for $j<k$, the $j^{\text {th }}$ derivative is the sum of all terms of form

$$
\sum(-1)^{m}(0,2)^{\alpha_{1}}(2,4)^{\alpha_{2}} \ldots(2 m-2,2 m)^{\alpha_{m}} P_{\chi}\left(C^{\perp}\left(\sigma^{2 m}\right)\right) .
$$


Here the $\alpha$ 's represent derivatives and

$$
\alpha_{1}+\ldots+\alpha_{m}=j .
$$

As above, the contribution from all terms involving each fixed set of $\alpha$ 's vanishes at $t=0$, as a consequence of the flatness of the normal cones. For $j=k$, the same holds for all such groups with the exception of (3.49).

Proposition 3.2 is crucial for showing that in the context of the convergence problem described in Sect. 0 , as $\eta \rightarrow 0$, the measures $R_{\eta}^{j}$ stay uniformly bounded.

We now wish to calculate the variational derivative of the analogs of the Lipschitz-Killing curvatures. Since our result follows from that of Regge, (2.28), it is not entirely local. Rather it involves something analogous to Stokes' theorem.

Lemma 3.3 (Generalized Regge). Let $X_{t}^{n}$ be a 1-parameter family of piecewise flat spaces. Then the variation of the total (interior plus boundary) Lipschitz-Killing curvature is given by

$$
\left(R^{j}\right)^{\prime}=\sum_{\sigma^{n-j}} P_{\chi}\left(C^{\perp}\left(\sigma^{n-j}\right)\right)\left|\sigma^{n-j}\right|^{\prime}
$$

Proof. In view of (3.45)

$$
\begin{aligned}
& \sum_{\sigma^{n-j}} P_{\chi}\left(C^{\perp}\left(\sigma^{n-j}\right)\right)^{\prime}\left|\sigma^{n-j}\right| \\
& \quad=-\sum_{\sigma^{n-j+2}}\left\{\sum_{\sigma^{n-j} \subset \sigma^{n-j+2}}(n-j, n-j+2)^{\prime}\left|\sigma^{n-j}\right|\right\} P_{\chi}\left(C^{\perp}\left(\sigma^{n-j+2}\right)\right) \\
& \quad=0
\end{aligned}
$$

since the term inside the curly brackets vanishes by $(2.27)$.

\section{Limiting Values of Face Angles}

We begin with the following model problem. Consider a geodesic triangle $\sigma_{t}^{2}$ in a 2-dimensional space of constant curvature $K$, with side lengths $t l_{i}, t l_{j}, t l_{k}$, where $\max \left(l_{i}, l_{j}, l_{k}\right)=1$. Let $\alpha_{i}(t), \alpha_{j}(t), \alpha_{k}(t)$ denote the corresponding angles. We wish to calculate $\lim _{t \rightarrow 0} \alpha_{i}(t)$ up to terms of order $t^{2}$, under the assumption that

$$
0<\alpha_{0}<\alpha_{i}(0)<\pi-\alpha_{0},
$$

for some $\alpha_{0}$. It will suffice to assume $K>0$, since the case $K<0$ is analogous and $K=0$ is trivial. Set $\sqrt{K} l_{i} t=s_{i}$. Then by the spherical law of cosines

$$
\begin{aligned}
\cos \alpha_{i}(t)= & \frac{\cos s_{i}-\cos s_{j} \cos s_{k}}{\sin s_{j} \sin s_{k}} \\
= & \frac{1}{s_{j} s_{k}}\left\{\left(1-s_{i}^{2} / 2+s_{i}^{4} / 24\right)-\left(1-s_{j}^{2} / 2+s_{j}^{4} / 24\right)\left(1-s_{k}^{2} / 2+s_{k}^{4} / 24\right)\right\} \\
& \cdot\left(1-s_{j}^{2} / 6\right)\left(1-s_{k}^{2} / 6\right)+O\left(s^{3}\right) .
\end{aligned}
$$


By the Euclidean law of cosines,

$$
\begin{gathered}
\cos \alpha_{i}(0)=\frac{s_{j}^{2}+s_{k}^{2}-s_{i}^{2}}{2 s_{j} s_{k}}, \\
\cos ^{2} \alpha_{i}(0)=\frac{s_{j}^{4}+s_{k}^{4}+s_{i}^{4}-2 s_{i}^{2}\left(s_{j}^{2}+s_{k}^{2}\right)+2 s_{j}^{2} s_{k}^{2}}{4 s_{j}^{2} s_{k}^{2}} .
\end{gathered}
$$

Using these relations, the right hand side of (4.2) simplifies to

$$
\cos \alpha_{i}(0)+\left(\cos ^{2} \alpha_{i}(0)-1\right) \frac{s_{j} s_{k}}{6}+O\left(s^{3}\right)=\cos \alpha_{i}(0)-\sin ^{2} \alpha_{i}(0) \frac{s_{j} s_{k}}{6}+O\left(s^{3}\right)
$$

Now if $\mathrm{a} \neq 1,-1$,

$$
\cos ^{-1} x=\cos ^{-1} a-\frac{1}{\sqrt{1-a^{2}}}(x-a)+c_{a}(x-a)^{2},
$$

where $c_{a}$ is a constant depending on $a$. Thus, by (4.1), if $a=\cos \alpha_{i}(0)$, and $x$ is the quantity in (4.6), we get

$$
\alpha_{i}(t)=\alpha_{i}(0)+\frac{1}{6} \sin \alpha_{i}(0) K l_{j} l_{k} t^{2}+O\left(K^{3 / 2} t^{3}\right) .
$$

Note that $\alpha_{i}(0)$ is the angle opposite the side of length $l_{i} t$, in the euclidean triangle $\sigma_{t, e}^{2}$ with sides of length $l_{i} t, l_{j} t, l_{k} t$. The area of this triangle is $\frac{1}{2} \sin \alpha_{i}(0) l_{j} l_{k} t^{2}$. By the Gauss-Bonnet formula for geodesic triangles

$$
\begin{aligned}
& \left(\alpha_{i}(t)-\alpha_{i}(0)\right)+\left(\alpha_{j}(t)-\alpha_{j}(0)\right)+\left(\alpha_{k}(t)-\alpha_{k}(0)\right) \\
& \quad=\alpha_{i}(t)+\alpha_{j}(t)+\alpha_{k}(t)-\pi \\
& =\int_{\sigma_{t}^{2}} K \cdot d A \\
& \quad=\frac{K}{2} \sin \alpha_{i}(0) l_{j} l_{k} t^{2}+O\left(K^{3 / 2} t^{3}\right)
\end{aligned}
$$

Thus, the content of (4.7) is that the angle differences, $\alpha(t)-\alpha(0)$ are apportioned equally among the three angles.

Now consider a geodesic triangle with sides $\gamma_{i}, \gamma_{j}, \gamma_{k}$ of length $l_{i} t, l_{j} t, l_{k} t$ in an arbitrary Riemannian manifold $M^{n}$. Let $K$ be the sectional curvature at the vertex $p_{i, t}$ opposite $\gamma_{i}$ of the plane section $\sigma$, spanned by the tangent vectors to $\gamma_{j}, \gamma_{k}$. Assume for convenience that the exponential map, $\exp _{p_{i, t}}$, is a diffeomorphism on a metric ball $B_{1}\left(p_{i, t}\right)$ of radius 1 , centered at $p_{i, t}$. Let $R$ denote the full curvature tensor of $M^{n}$ and $\nabla R$ its covariant derivative. Assume that

$$
\sup _{B_{1}\left(p_{i, t}\right)} \max (\|R\|,\|\nabla R\|) \leqq 1 .
$$

In the general case, these normalizations can be achieved by rescaling the metric. In Appendix A2 we show that the estimate (4.7) continues to hold (with the constant in the error term $O\left(t^{3}\right)$ controlled by the geometric bounds we have assumed). 


\section{Statement of Main Theorem and Strategy of Proof}

We now come to the main theorem (concerning the interior term) to be proved in this work. ${ }^{5}$ Let $M^{n}$ be a Riemannian manifold and let $M_{\eta}^{n}$ be a p.l. space of mesh $\eta$ obtained from a smooth triangulation of $M^{n}$ and the resulting geodesic distances (see Sect. 1). For any open, bounded set $U$ let

$$
U \rightarrow R^{j}(U)
$$

be the signed measure obtained by integrating the $j^{\text {th }}$ Lipschitz-Killing curvature form of $M^{n}$ over $U$. For given $M_{\eta}^{n}$, we define another signed measure

$$
U \rightarrow R_{\eta}^{j}(U)
$$

as follows. By the equivalent formulas (3.23), (3.37), and (3.38) the combinatorial $j^{\text {th }}$ Lipschitz-Killing curvature for $M^{n}$ is a sum of combinations $P_{\chi}\left(C^{\perp}\left(\sigma^{n-j}\right)\right)$ from each $n-j$ simplex $\sigma^{n-j}$. Define $R_{\eta}^{j}(U)$ to be

$$
\sum P_{\chi}\left(C^{\perp}\left(\sigma^{n-j}\right)\right) \operatorname{Vol}\left(\sigma^{n-j} \cap U\right) .
$$

In the statement of Theorem 5.1, we will assume that a given $U$ has a regular boundary in the following sense: The volume $\left|T_{r}(\partial U)\right|$ of the set $T_{r}(\partial U)$ of points of distance less than $r$ to $\partial U$ tends to zero with $r$. With these conventions, we have

Theorem 5.1. There is $c=c(\|R\|,\|\nabla R\|, \Theta)$ and $\eta_{0}=\eta_{0}(\|R\|,\|\nabla R\|, \Theta)>0$, such that

$$
\left|R_{\eta}^{j}(U)-R^{j}(U)\right| \leqq c\left(|U| \sqrt{\eta}+\left|T_{\sqrt{\eta}}(\partial U)\right|\right)
$$

whenever $\eta \leqq \eta_{0}$ and $M_{\eta}^{n}$ has fatness $\geqq \Theta$. In particular, as $\eta$ tends to zero, $R_{\eta}^{j}(U)$ converges to $R^{j}(U)$, whenever $\left\{M_{\eta}^{n}\right\}$ is a family with fatness bounded away from zero.

We will begin by explaining Theorem 5.1 in the 2-dimensional case. Not surprisingly, this is a straightforward consequence of the Gauss-Bonnet formula for geodesic triangles. It follows even more directly from (4.7). Let $M_{\eta}^{2}$ be a piecewise flat approximation to the triangulated Riemannian manifold $M^{2}$. Let the fatness be $\Theta$. If $\alpha$ is an angle of $\sigma^{2}$, let $\alpha_{\eta}$ denote the corresponding angle of $\sigma_{\eta}^{2}$. Since for each $\sigma^{0}$

$$
\sum_{\sigma^{2} \supset \sigma^{0}} \alpha=2 \pi
$$

we obtain from (4.7)

$$
R_{\eta}^{2}\left(\sigma^{0}\right)=\frac{1}{3} R\left(\sigma^{0}\right) \cdot\left|s t\left(\sigma^{0}\right)\right|+O\left(\eta^{3}\right) .
$$

If $U \subset M^{2}$ is an open set with smooth boundary, summing over $\sigma^{0} \subset U$ easily gives

$$
R_{\eta}^{2}(U)=R^{2}(U)+O(\eta)
$$

It is tempting to explore the possibility of generalizing the above argument directly to higher dimensions (see Appendix 1 for further discussion). Instead we will proceed as follows.

5 In Sect. 8, we show how to modify our arguments to obtain the analogous result for the boundary curvatures, $H^{j}, H_{\eta}^{j}$ 
Consider first the case $R^{j}\left(M^{j}\right)$. Fix a vertex $\sigma^{0}$ of our triangulation, and let $L$ denote the induced triangulation of $S^{j-1} \subset M_{\sigma^{0}}$ by totally geodesic simplices. As in Proposition 3.2 , we can consider the function $R^{j}(C(\mathscr{L})$ ) on the space of all piecewise flat cones $C(\mathscr{L})$, having the same combinatorial structure as $C(L)$. Coordinates on this space can be taken as edge lengths of the spherical complex $L$, or in notation of Sect. 3 , the angles denoted by $(0,2)$. According to Proposition 3.2, the function $R^{j}(C(\mathscr{L}))$ has a critical point of order $(j / 2-1)$ at the flat cone $C(L)$. Moreover, let $C\left(L_{\eta}\right)$ denote the nearby piecewise flat cone obtained from $M_{\eta}^{j}$ at the vertex $\sigma_{\eta}^{0}$ correspodning to $\sigma^{0}$. Then $R^{j}\left(C\left(L_{\eta}\right)\right)$ can be estimated by Taylor's theorem. For each angle $(0,2)$ at $\sigma^{0}$, set

$$
(0,2)_{t}=(0,2)+t\left\{(0,2)_{\eta}-(0,2)\right\} .
$$

The $(0,2)_{t}$ determine a 1-parameter family $L(t)$ with $L(0)=L$ and $L(1)=L_{\eta}$. Each pair of faces $\tau_{t}^{2 k-3} C \tau_{t}^{2 k-1} C L(t)$ determines a dihedral angle $(2 k-2,2 k)_{t}$. The derivative $(2 k-2,2 k)_{t}^{\prime}$ is given explicitly by (A1.17) (with $n$ replaced by $2 k$ ). For our purposes it is enough to note that

$$
(2 k-2,2 k)_{t}^{\prime}=\sum_{l=1}^{\left(\begin{array}{c}
2 k-1 \\
2
\end{array}\right)} f_{l}\left(\left(0, \sigma_{1}^{2}\right)_{t}, \ldots,\left(0, \sigma_{\left.\left.\left(\begin{array}{c}
2 k-1 \\
2
\end{array}\right)\right)_{t}\right)}^{2}\left(0, \sigma_{l}^{2}\right)^{\prime},\right.\right.
$$

where the $\left(0, \sigma_{l}^{2}\right)_{t}$ correspond to the edges $\tau_{l}^{1}$ of $\tau^{2 k-1}$ and the coefficients $f_{l}$ are smooth and bounded by some $c(\Theta)$. Thus the expression in (3.48) of Proposition 3.2 can be regarded as a certain polynomial of degree $j / 2$ in the $(0,2)^{\prime}=(0,2)_{\eta}$ $-(0,2)$, whose coefficients are smooth bounded functions of the $(0,2)$ for fatness $>\Theta>0$. But by (4.7) and (A2.2)

$$
\left|\left(0, \sigma_{l}^{2}\right)^{\prime}-\frac{1}{3} K_{l}\right| \sigma_{l}^{2}|| \leqq c(\|R\|,\|\nabla R\|, \Theta) \eta^{3},
$$

where $\sigma_{l}^{2}$ is a 2-simplex at $\sigma^{0}\left(\left|\sigma_{l}^{2}\right| \leqq c \eta^{2}\right)$ and $K_{l}$ the sectional curvature at $\sigma^{0}$ of the plane spanned by the corresponding geodesic directions.

Applying (5.9), (5.10), Proposition 5.2 and Taylor's Theorem in the form (A2.11), we obtain

Proposition 5.2. There is an estimate

$$
\begin{aligned}
& \mid R^{j}\left(C\left(L_{\eta}\right)\right)-P_{j}\left(\left(0, \sigma_{1}^{2}\right), \ldots,\left(0, \sigma_{(2 k-1)}^{2}\right), \frac{K_{1}}{3}\left|\sigma_{1}^{2}\right|, \ldots, \frac{K_{\left(\begin{array}{c}
2 k-1 \\
2
\end{array}\right)}}{3}\left|\sigma_{\left(\begin{array}{c}
2 k-1 \\
2
\end{array}\right)}^{2}\right|\right) \\
& \quad \leqq c(\|R\|,\|\nabla R\|, \Theta) \eta^{j+1},
\end{aligned}
$$

where $P_{j}$ is a polynomial in the $K_{l}\left|\sigma_{l}^{2}\right|$ with coefficients which are smooth functions of the $\left(0, \sigma_{l}^{2}\right)$ for fatness $>\Theta$. Moreover, the function $P_{j}$ depends only on the combinatorial structure of $L$.

Let $D^{j}\left(\sigma^{0}\right)$ denote the "dual cell" to $\sigma^{0}$, the set of points closer to $\sigma^{0}$ than any other vertex. Let $\left|D^{j}\left(\sigma^{0}\right)\right|$ denote its volume and $* R^{j}\left(\sigma^{0}\right)$ the Chern-Gauss-Bonnet density of $M^{j}$ (j even) at $\sigma^{0}$. If it so happened for example that the polynomial $P_{j}$ satisfied

$$
\left|\frac{P_{j}}{\left|D^{j}\left(\sigma^{0}\right)\right|}-* R^{j}\left(\sigma^{0}\right)\right|=o(1)
$$


our main result would follow. But this is not the case. What we can say is that $P_{j} /\left|D^{j}\left(\sigma^{0}\right)\right|$ is a polynomial of degree $j / 2$ in the curvature tensor $R$ at $\sigma^{0}$, whose coefficients are bounded in terms of $\Theta$. In order to arrive at $R^{j}$ from $P_{j}$, we must add the contributions of many different vertices. Specifically we consider a normal coordinate ball $B_{r}(p)$, such that $r$ and $\eta / r$ both are small. The portion of our triangulation $T$ lying in $B_{r}(p)$ induces a triangulation $\tilde{T}$ of a subregion of $B_{r}(0)$ $C M_{p}$, the vertices of which are the inverse images $\exp _{p}^{-1}\left(\sigma^{0}\right)$ of the vertices of $T$. The map $\exp _{p}$ also induces an identification of $M_{\sigma_{\alpha}}^{j}$ with $M_{p}^{j}$ for each vertex $\sigma_{\alpha}^{0} \subset B_{r}(p)$. If we replace the sectional curvatures which are substituted into $P_{j}$ at $\sigma_{\alpha}^{0}$ by their corresponding values at $p$ and the values of the coefficients by their corresponding values for $\tilde{T}$, we introduce an error which is $\leqq c(\|R\|,\|\nabla R\|, \Theta) r \eta^{j}$. Call the resulting polynomial $P^{j}\left(\sigma_{\alpha}^{0}, p\right)$ and set

$$
\mathscr{P}^{j}(r, \tilde{T})=\left|B_{r}(p)\right|^{-1} \sum_{\sigma_{\alpha}^{0} C B_{r}(p)} P^{j}\left(\sigma_{\alpha}^{0}, p\right) .
$$

Here the notation $\mathscr{P}^{j}(r, \tilde{T})$ indicates that $\mathscr{P}^{j}$ depends on $r$ and the given triangulation. Since

$$
\#\left(\sigma_{\alpha}^{0} C B_{r}(p)\right) \leqq c(\Theta)\left|B_{r}(p)\right| \eta^{-n},
$$

estimate (5.13) and our arguments combine to give

$$
\left.\left|\mathscr{P}^{j}(r, \tilde{T})-\right| B_{r}(p)\right|^{-1} R_{\eta}^{j}\left(B_{r}(p)\right) \mid \leqq c(\|R\|,\|\nabla R\|, \Theta)(\eta+r) .
$$

Note that the orthogonal group of $M_{p}^{n}$ acts on polynomials in $R$ and also on the lifts via $\exp _{p}^{-1}$ of triangulations of subsets of $B_{r}(p)$. It is an immediate consequence of construction that

$$
g^{j}(r, \tilde{T})=\mathscr{P}^{j}(r, g \tilde{T})
$$

for $g$ in the orthogonal group of $M_{p}^{n}$.

We can now state the basic assertion of this section.

Proposition 5.3. There is an estimate

$$
\left|\mathscr{P}^{j}(r, \tilde{T})-* R^{j}(p)\right| \leqq c\left(\frac{\eta}{r}+r\right),
$$

where $c=c(\|R\|,\|\nabla R\|, \Theta)<\infty$ and $\Theta$ is the fatness of the triangulation.

It is essentially obvious that (5.17) implies our main result, Theorem 5.1, by choosing $r=\sqrt{\eta}$ (see Lemma 6.2). Here we reduce the verification of (5.17) to the statement that $\lim _{\substack{r \rightarrow 0 \\ \eta / r \rightarrow 0}} \mathscr{P}^{j}(r, \tilde{T})$ exists and is independent of the particular sequence of triangulations used, as long as the fatness stays bounded away from zero.

Lemma 5.4. Let $T_{1}, T_{2}$ be triangulations of $M^{j}$ as above. The estimate (5.17) is implied by the estimate that for all such $T_{1}, T_{2}$ with mesh $\eta_{1}$ and $\eta_{2}$ and fatness $\geqq \Theta>0$, there is a $c=c(\|R\|,\|\nabla R\|, \Theta)<\infty$ such that

$$
\left|\mathscr{P}^{j}\left(r, \tilde{T}_{1}\right)-\mathscr{P P}^{j}\left(r, \tilde{T}_{2}\right)\right| \leqq c\left(\frac{\eta_{1}+\eta_{2}}{r}+r\right) .
$$


Proof. Since the discussion is local, it suffices to restrict attention to metrics on euclidean space $E^{j}$. Let $R$ be a curvature tensor at the tangent space $E^{j}$ to the origin $0 \subset E^{j}$. Let $\left\{g_{R}\right\}$ denote the set $g_{R}$ of Riemannian metrics whose curvature tensor at $0 \in E^{j}$ is $R$. Then for each $R,\left\{g_{R}\right\}$ is nonempty. Thus we obtain a polynomial $R \rightarrow \mathscr{P}^{j}(R, r, \tilde{T})$. Since the $\mathscr{P}^{j}(r, T)$ are bounded independent of $T$ (the fatness is bounded away from zero), it follows from (5.18) that $\lim _{\substack{r \rightarrow 0 \\ \eta / r \rightarrow 0}} \mathscr{P}^{j}(r, \tilde{T})$ exists.

If we take $T_{1}=\hat{T}, T_{2}=g T$ in (5.18), and apply (5.17) it follows that this limit is an invariant polynomial in $R$. Moreover, if the metric $g$ on $B_{r}(0)$ splits isometrically as $M^{j-1} \times \mathbb{R}$, then by taking $T$ of the form $T=T_{1} \times T_{2}$ and using Corollary 3.1, we see that $\mathscr{P}^{j}(r, \tilde{T})$ vanishes. Similarly, let $g=h \times \ldots \times h$, with $j / 2$ factors which are 2-dimensional of constant curvature, and let $T$ be of the form $T=S \times \ldots \times S(j / 2$ factors). By use of (3.34) and the 2-dimensional case verified at the beginning of this section, an obvious calculation shows that $\lim _{\substack{r \rightarrow 0 \\ \eta / r \rightarrow 0}} \mathscr{P}^{j}(r, \tilde{T})=* R^{j}$ for such $g$ and $T$.

According to the result of Gilkey [G1], these three properties characterize the Chern-Gauss-Bonnet form. So the proof is complete.

Observe that the definition of the polynomial $\mathscr{P}^{j}(r, \tilde{T})$, and the rest of the discussion generalize immediately to the case $R^{j}\left(M^{n}\right)$. The normal cone to a simplex $\sigma^{n-j}$ becomes well defined once one picks a vertex $\sigma^{0} C \sigma^{n-j}$. Thus, as a replacement for $C(L)$ one can simply use the normal cones corresponding to all of the vertices of $\sigma^{n-j}$ and average the resulting polynomials to form $\mathscr{P}^{j}(r, \tilde{T})$. The contribution due to each normal case is $O\left(\eta^{j}\right)$ and this is multiplied by $\left|\sigma^{n-j}\right|=O\left(\eta^{n-j}\right)$. To generalize Proposition 5.4 one uses the full statement of Gilkey's theorem for arbitrary $j$.

To close this section, we note the following important bound on the measure $R_{\eta}^{j}$.

Lemma 5.5. Let $U \subset M$. Then

$$
\left|R_{\eta}^{j}(U)\right| \leqq c\|R\|^{j / 2}\left|T_{\eta}(U)\right|,
$$

where $T_{\eta}(U)=\{x \mid \operatorname{dist}(x, U) \leqq \eta\}$ and $c=c(\|R\|,\|\nabla R\|, \Theta)$.

Proof. The contribution to $R_{\eta}^{j}(U)$ coming from any particular $\sigma^{n-j} \subset U$ is bounded by $c(\|R\|,\|\nabla R\|, \Theta)\|R\|^{j / 2} \eta^{n}$ since

$$
\left|(0,2)_{\eta}-(0,2)\right| \leqq c(\|R\|,\|\nabla R\|, \Theta)\|R\| \eta^{2}
$$

by our previous arguments. The number of such simplices in $U$ is certainly bounded above by $\left(\begin{array}{c}n+1 \\ n-j+1\end{array}\right)$ times the number of $\sigma^{n}$ in $T_{\eta}(U)$. The latter is bounded by $c(\Theta) \eta^{-n}\left|T_{\eta}(U)\right|$, which gives (5.19).

\section{Proof Modulo Lemma on Triangulations}

In the previous section we associated a polynomial $\mathscr{P}^{j}(r, \tilde{T})$ of degree $j / 2$ in curvature to a real number $r$ and triangulation $T$ of mesh $\eta$ of the ball $B_{r}(p)$. 
According to Lemma 5.3, the fact that $\lim _{\substack{r \rightarrow 0 \\ \eta / r \rightarrow 0}} \mathscr{P}^{j}(r, \tilde{T})=* R^{j}$ follows from the statement that $\lim _{\substack{r \rightarrow 0 \\ \eta / r \rightarrow 0}} \mathscr{P}^{j}(r, \tilde{T})$ exists independent of the particular choice of $T$. We begin this section by recalling the (quite standard) argument by which the above equality implies our main result. We then proceed to establish the hypothesis of Lemma 5.3 modulo a lemma on triangulations (Lemma 6.3) to be proved in Sect. 7.

Lemma 6.1. Let $M^{n}$ be a Riemannian manifold with $\|R\|<1$. Let $\mu$ be a (signed) measure on $M^{n}$ such that for some $r$ less than the injectivity radius of $M^{n}$

$$
f_{\mu, r}(x)=\mu\left(B_{r}(x)\right) /\left|B_{r}(x)\right|,
$$

defines an integrable function with respect to the Riemannian measure $\omega$. Suppose

$$
\left|f_{\mu, r}(x)\right|<c .
$$

Let $U \subset M^{n}$ be contained in a compact set and let $\left|T_{s}(\partial U)\right|$ denote the volume of the set of points $T_{s}(\partial U)$ at distance $\leqq s$ from $\partial U$. Then there is a constant $c(n)$ such that

$$
\left|\int_{U} f_{\mu, r}(x) \omega-\mu(U)\right| \leqq c(n)\left(r^{2}[c|U|+\mu(U)]+c\left(1+c r^{2}\right)\left|T_{r}(\partial U)\right|\right) .
$$

Proof. Define subsets $S_{1}, S_{2}, \mathscr{V} \subset M \times M$ by

$$
\begin{gathered}
S_{1}=\left\{\left(x_{1}, x_{2}\right) \mid x_{1} \in U, x_{2} \in B_{r}\left(x_{1}\right)\right\}, \\
S_{2}=\left\{\left(x_{1}, x_{2}\right) \mid x_{2} \in U, x_{1} \in B_{r}\left(x_{2}\right)\right\}, \\
\mathscr{V}=\left\{\left(x_{1}, x_{2}\right) \mid x_{1} \in T_{r}(\partial U), x_{2} \in B_{r}\left(x_{1}\right)\right\} .
\end{gathered}
$$

Then

$$
\left(S_{1} \backslash S_{2}\right) \cup\left(S_{2} \backslash S_{1}\right) \subset \mathscr{V} .
$$

Consider the measure $\mu \times \omega$ on $M \times M$. Note that by (A2.6) (or by Rauch's comparison theorem, see $[\mathrm{CE}]$ )

$$
\left|\frac{B_{r}(x)}{V_{r}}-1\right| \leqq c(n) r^{2},
$$

where $V_{r}$ denotes the volume of the ball $B_{r}(0) \subset E^{n}$. Then by Fubini's theorem,

$$
\begin{gathered}
\frac{1}{V_{r}} \mu \times \omega\left(S_{1}\right)=\int_{U} \frac{\left|B_{r}(x)\right|}{V_{r}} d \mu=\mu(U)\left(1 \pm c(n) r^{2}\right), \\
\frac{1}{V_{r}} \mu \times \omega\left(S_{2}\right)=\int_{U} f_{\mu, r}(x) \frac{\left|B_{r}(x)\right|}{V_{r}} d \omega=\int_{U} f_{\mu, r} d \omega \pm c \cdot c(n) r^{2}|U|, \\
\frac{1}{V_{r}} \mu \times \omega(\mathscr{V}) \leqq c\left|T_{r}(\partial U)\right|\left(1+c(n) r^{2}\right) .
\end{gathered}
$$

Combining (6.7)-(6.9) completes the proof. 
Corollary 6.2. Theorem 5.1 is a consequence of (5.17).

Proof. Take first $\mu=R_{\eta}^{j}(\eta<r)$ in Lemma 6.1. By Lemma 5.4 the term $\mu(U)$ on the right hand side of estimate (6.3) may be bounded by $c(\|R\|,\|\nabla R\|, \Theta)|U|$ independent of $\eta$. Therefore

$$
\left|\int_{U} f_{R_{\eta}^{j}, r} \omega-R_{\eta}^{j}(U)\right| \leqq c(\|R\|,\|\nabla R\|, \Theta)\left(|U| r^{2}+\left|T_{r}(\partial U)\right|\right) .
$$

Similarly, we can take $\mu=R^{j}$ and we first recover the obvious result that

$$
\left|\int_{U} f_{R^{j}, r} \omega-\int_{U} R^{j}\right| \leqq c(\|R\|,\|\nabla R\|, \Theta)\left(|U| r^{2}+\left|T_{r}(\partial U)\right|\right) .
$$

Now (5.17) reformulated in terms of the quantities just introduced easily gives

$$
\left|f_{R_{\eta}^{j}, r}(x)-f_{R^{j}, r}(x)\right| \leqq c(\|R\|,\|\nabla R\|, \Theta)\left(\frac{\eta}{r}+r\right) .
$$

Combining (6.12) with the estimates (6.10) and (6.11) just stated, proves Corollary 6.2 , if we take $r=\eta^{1 / 2}$.

We can now proceed in two steps. In doing so, we will formulate everything in the tangent space $E_{0}^{n}$ at $p$. We start with a basic lemma on triangulations of $B_{r}(0)=\exp _{p}^{-1} B_{r}(p) \subset E_{0}^{n}$.

Lemma 6.3. Let $\tilde{T}_{1}$ and $\tilde{T}_{2}$ be two triangulations of subsets of $E_{0}^{n}$ containing $B_{r}(0)$ of mesh $\tilde{\eta}_{1}, \tilde{\eta}_{2}\left(\tilde{\eta}_{1} \leqq \tilde{\eta}_{2}\right)$, respectively, and fatness $\geqq \tilde{\Theta}>0$. Then there exists $c(\tilde{\Theta})>0$ and triangulations $\tilde{T}_{3}$ and $\tilde{T}_{4}$ of open subsets of $B_{r}(0)$ of mesh $\leqq \frac{3}{2} \tilde{\eta}_{1}$ and $\tilde{\eta}_{2}$, respectively, and fatness $\geqq c(\widetilde{\Theta})$ such that

(i) $\tilde{T}_{l}=\tilde{T}_{l+2}(l=1,2)$ on $B_{r-8 \tilde{\eta}_{2}}(0)$,

(ii) $\tilde{T}_{3}$ and $\tilde{T}_{4}$ agree near their common boundary.

Granted Lemma 6.3, the proof of Theorem 5.1, can now be completed for the case of the Gauss-Bonnet form $R^{n}\left(M^{n}\right)$. By our previous discussion (Lemma 6.2), it suffices to establish (5.18):

Proof of Estimate (5.18) $(j=n)$. Let $T_{1}$ and $T_{2}$ be triangulations of $M^{n}$ of mesh $\eta_{1}, \eta_{2}\left(\eta_{1} \leqq \eta_{2}\right)$, respectively, and fatness $\Theta>0$. Let $\tilde{T}_{l}=\exp _{p}^{-1} T_{l}(l=1,2)$ be their lifts to the tangent space of $p$. By (1.22) there is easily seen to be $c=c(\|R\|,\|\nabla R\|, \Theta)<\infty$ such that their meshes $\tilde{\eta}_{1}, \tilde{\eta}_{2}\left(\tilde{\eta}_{1} \leqq \tilde{\eta}_{2}\right)$ and fatness $\tilde{\Theta}$ satisfy

$$
\tilde{\eta}_{l} \leqq c \eta_{l}, \quad \tilde{\Theta} \geqq c^{-1} \Theta
$$

whenever $r \leqq 1$. By Lemma 6.3 we can modify these on $B_{r}(0) \backslash B_{r-8 \eta_{2}}(0)$ to obtain $\tilde{T}_{3}, \tilde{T}_{4}$ which agree near their common boundary in $B_{r}(0)$. By the piecewise flat Chern-Gauss-Bonnet formula, [see (3.7), (3.8), (3.10)], we have in particular

$$
R^{n}\left(\tilde{T}_{3}\right)+H^{n}\left(\partial \tilde{T}_{3}\right)=\mathrm{const}=R^{n}\left(\tilde{T}_{4}\right)+H^{n}\left(\partial \tilde{T}_{4}\right),
$$

which implies

$$
R^{n}\left(\tilde{T}_{3}\right)=R^{n}\left(\tilde{T}_{4}\right)
$$


since $\tilde{T}_{3}=\tilde{T}_{4}$ near $\partial \tilde{T}_{3}=\partial \tilde{T}_{4}$. But by Lemma 5.4

$$
\left|R^{n}\left(\tilde{T}_{l}\right)-R^{n}\left(\tilde{T}_{l+2}\right)\right| \leqq c(\|R\|,\|\nabla R\|, \Theta) r^{n} \frac{\eta}{r}, \quad l=1,2
$$

which suffices to complete the proof.

Remark 6.1. In view of the fact that for $M^{n}$ closed $R^{n}\left(M^{n}\right)=\chi\left(M^{n}\right)$, Theorem 5.1 yields a new proof of the Chern-Gauss-Bonnet formula for closed smooth manifolds (the results of Sect. 8 yield the formula for smooth manifolds with boundary).

In order to generalize the above argument to give a proof of (5.18) for $j \neq n$ ( $j$ even) we need a replacement for (6.13), the topological invariance of $R^{n}+H^{n}$. The appropriate replacement turns out to be Lemma 3.3 (the generalized Regge lemma). In fact, if $j=n,(3.52)$ of Lemma 3.3 simply reduces to the statement that $R^{n}+H^{n}$ is an invariant, independent of the piecewise flat metric.

Proof of Estimate (5.19) ( $j$ Arbitrary). Let $g$ denote the Riemannian metric on $B_{r}(p)$ and let $\hat{g}$ denote the induced flat metric at $E_{0}^{n}$, the tangent space at $p$. Let $D_{t}: E_{0}^{n} \rightarrow E_{0}^{n}$ denote dilation by $t$ and define $g_{t}$ by

$$
g_{t}=\left(\exp _{p}^{-1}\right)^{*}\left\{\frac{1}{t^{2}} D_{t}^{*} \exp _{p}^{*} g\right\} .
$$

Then $g_{1}=g$ and $g_{t}$ extends smoothly to $[0,1]$ in such a way that $g_{0}=\left(\exp _{p}^{-1}\right)^{*} \hat{g}$. By construction, the curvature $R(t)$ of $g_{t}$ satisfies

$$
\begin{gathered}
\|R(t)\| \leqq\|R\|, \\
\|\nabla(t) R(t)\| \leqq\|\nabla R\|,
\end{gathered}
$$

where $R$ is the curvature of $g$. Furthermore for given $\Theta$ and $r \leqq r_{0}(\|R\|, \Theta)$, if the triangulation $T$ defines a p.l. space with geodesic distances defined by $g$ and of mesh $\eta$ and fatness $\geqq \Theta$ inside $B_{r}(0)$, then the p.l. space obtained by replacing $g$ by $g_{t}$ has mesh $\eta(t)$ inside $B_{r}(p)$ satisfying

$$
\eta(t) \leqq 2 \eta
$$

Let $T_{1}$ and $T_{2}$ be as above and choose $\tilde{T}_{3}$ and $\tilde{T}_{4}$ again in the same way. Employing Lemma 5.4 once again, it suffices to establish $(5.18)$ for $\tilde{T}_{3}, \tilde{T}_{4}$. Let $T_{l}(t)(l=3,4)$ denote the piecewise flat approximation for the geodesic triangulation with respect to $g_{t}$ and having the same vertices as $\exp _{0}\left(\tilde{T}_{l}\right)$. Note that near the boundary $T_{3}(t)$ and $T_{4}(t)$ coincide for all $t$ and for $t=0$, both are flat in the interior. Thus

$$
\begin{aligned}
R^{j}\left(T_{3}(1)\right)-R^{j}\left(T_{4}(1)\right)= & {\left[R^{j}\left(T_{3}(1)\right)+H^{j}\left(T_{3}(1)\right)-R^{j}\left(T_{3}(0)\right)-H^{j}\left(T_{3}(0)\right)\right] } \\
& -\left[R^{j}\left(T_{4}(1)\right)+H^{j}\left(T_{4}(1)\right)-R^{j}\left(T_{4}(0)\right)-H^{j}\left(T_{4}(0)\right)\right]
\end{aligned}
$$

where we have used

$$
R^{j}\left(T_{3}(0)\right)=R^{j}\left(T_{4}(0)\right)
$$


and

$$
H^{j}\left(T_{3}(t)\right)=H^{j}\left(T_{4}(t)\right)
$$

By (3.52)

$$
\begin{gathered}
R^{j}\left(T_{l}(1)\right)+H^{j}\left(T_{l}(1)\right)-R^{j}\left(T_{l}(0)\right)-H^{j}\left(T_{l}(0)\right) \\
\quad=\int_{0}^{1} \sum_{\sigma^{n-j}} P_{\chi}\left(C^{\perp}\left(\sigma_{t}^{n-j}\right)\right)\left|\sigma_{t}^{n-j}\right|^{\prime} d t .
\end{gathered}
$$

As in Sect. 5, for $0 \leqq t \leqq 1$

$$
\left|R^{j}\left(C^{\perp}\left(\sigma_{t}^{n-j}\right)\right)\right| \leqq c(\Theta)\|R(t)\|^{j / 2} \eta^{j}(t) \leqq 2^{j} c(\Theta)\|R\|^{j / 2} \eta^{j},
$$

where the last estimate follows from (6.17) and (6.18). Moreover, since (6.16) gives

$$
g_{t}=\exp _{p}^{*}\left(\hat{g}+A(t) r^{2}+B(t, r) r^{3}\right)
$$

where $\|A(t)\|,\|B(t, r)\|$ can be bounded by $\|R(t)\|$ and $\|\nabla(t) R(t)\|$ respectively, it follows easily that

$$
\left|\sigma_{t}^{n-j}\right|^{\prime} \leqq c \eta^{n-j} r^{2}
$$

The crucial point is that $\exp ^{-1} \mid B_{r}(p) \subset E_{0}^{n}$ preserves lengths up to a factor $\left(1+O\left(r^{2}\right)\right)$. Combining (6.19), (6.22), (6.23), and (6.25) gives

$$
\frac{1}{\left|B_{r}(p)\right|}\left|R^{j}\left(T_{3}(1)\right)-R^{j}\left(T_{4}(1)\right)\right| \leqq c(\Theta)\|R\|^{j / 2} r^{2},
$$

which suffices to complete the proof.

Remark 6.2. Note that for each individual $\sigma^{n-j}$ we have only

$$
\left|P_{\chi}^{j}\left(C^{\perp}\left(\sigma_{t}^{n-j}\right)\right)^{\prime}\right| \sigma_{t}^{n-j}|| \leqq C\|R\|^{j / 2} \eta^{j} \eta^{n-j},
$$

which, after dividing by $\left|B_{r}(p)\right|$, is not negligible. Thus it seems that the generalized Regge lemma (4.43) is definitely required to show that the total contribution due to these terms vanishes.

\section{Lemma on Triangulations}

In this section we prove the lemma on triangulations as stated in Sect. 6, Lemma 6.3. The proof uses only elementary facts about general position of subspaces of $E^{n}$. The main point is to do the constructions in such a way that they are independent of the number of vertices (i.e. so that the effect of moving many vertices does not become cumulative). We begin with some definitions. Let $\mathscr{E}_{1}^{k_{1}}, \mathscr{E}_{2}^{k_{2}} C E^{n}$ be affine subspaces and let $\tilde{\mathscr{E}}_{1}^{k_{1}}, \tilde{\mathscr{E}}_{2}^{k_{2}}$ be the parallel subspaces through the origin, $0 \in E^{n}$. Write $\mathscr{E}_{1}^{k_{1}} \pitchfork_{\delta} \mathscr{E}_{2}^{k_{2}}\left(\mathscr{E}_{k} k_{1}, \mathscr{E}_{2}^{k_{2}}\right.$ are $\delta$-transverse) if the following holds
A)
$\operatorname{dim}\left(\tilde{\mathscr{E}}_{1}^{k_{1}} \cap \tilde{\mathscr{E}} k_{2}\right)=\max \left(0, k_{1}+k_{2}-n\right)$,
B)
$0<\delta<\Varangle\left(\tilde{\mathscr{E}}_{1}^{k_{1}}, \tilde{\mathscr{E}}_{2}^{k_{2}}\right)=\Varangle\left(\mathscr{E}_{1}^{k_{1}}, \mathscr{E}_{2}^{k_{2}}\right)$,
C)
$\operatorname{dist}\left(\mathscr{E}_{1}^{k_{1}}, \mathscr{E}_{2}^{k_{2}}\right)>\delta, \quad$ if $\quad k_{1}+k_{2}<n$. 
Here

$$
\Varangle\left(\tilde{\mathscr{E}}_{1}^{k_{1}}, \tilde{\mathscr{E}}_{2}^{k_{2}}\right)=\min _{\left(e_{1}, e_{2}\right)} \cos ^{-1}\left(e_{1}, e_{2}\right),
$$

where $\left\|e_{j}\right\|=1, e_{j} \in\left(\tilde{\mathscr{E}} k_{1} \cap \tilde{\mathscr{E}}{ }_{2}\right)^{\perp} \cap \tilde{\mathscr{E}}_{j}^{k_{j}}$.

Now let $\sigma^{k} C E^{n}$ denote an open simplex with closure $\bar{\sigma}^{k}=\sigma^{k} \cup \partial \sigma^{k}$.

Let $[A]$ denote the affine subspace generated by the points in the set $A \subset E^{n}$. Suppose $\sigma_{j}^{k_{j}}$ has mesh $\eta_{j}, j=1,2$ and $\eta_{1} \leqq \eta_{2}$. Write $\sigma_{1}^{k_{1}} \pitchfork_{\delta} \sigma_{2}^{k_{2}}$ if

$$
\begin{aligned}
& \operatorname{dim}\left(\left[\tilde{\sigma}_{1}^{k_{1}}\right]\right.\left.\cap\left[\tilde{\sigma}_{2}^{k_{2}}\right]\right)=\max \left(0, k_{1}+k_{2}-n\right), \\
& 0<\delta<\Varangle\left(\left[\sigma_{1}^{k_{1}}\right],\left[\sigma_{2}^{k_{2}}\right]\right), \\
& \operatorname{dist}\left(\sigma_{1}^{l_{1}}, \sigma_{2}^{l_{2}}\right)>\delta \cdot \eta_{1},
\end{aligned}
$$

whenever $\sigma_{j}^{l_{j}} \subset \bar{\sigma}_{j}^{k_{j}}$ and $l_{1}+l_{2}<n$.

The intersection $\bar{C}=\bar{\sigma}^{k_{1}} \cap \bar{\sigma}^{k_{2}}$ of arbitrary closed simplices is a closed, convex polyhedral cell. To obtain a triangulation of $\bar{C}$, we can form the first barycentric subdivision $\bar{C}^{\prime}$ as follows. For each face $\bar{C}_{\alpha} \subset \partial \bar{C}$ choose an interior point $p_{\alpha} \in C_{\alpha} \subset \bar{C}_{\alpha}$. The simplices of $C^{\prime}$ are spanned by all sets $p_{\alpha_{1}}, \ldots, p_{\alpha_{t}}$, where

$$
\bar{C}_{\alpha_{i}} \subset \bar{C}_{\alpha_{i+1}} \quad(i=1, \ldots, t-1) .
$$

The following lemma controls the fatness of $\bar{C}^{\prime}$.

Lemma 7.1. Given $\Theta, \eta, \delta>0$, there exists $c(k, \Theta, \delta)>0$ with the following properties. Let $\sigma_{1}^{k_{1}}, \sigma_{2}^{k_{2}} \subset E^{k}, k=\max \left(k_{1}, k_{2}\right)$ have fatness $\geqq \Theta$ and mesh $\eta_{1}$ and $\eta_{2}$, respectively, with $\eta_{1} \leqq \eta_{2}$. Assume further that $\sigma_{1}^{k_{1}} \pitchfork_{\delta} \sigma_{2}^{k_{2}}$.

(1) Then if $\sigma^{l_{j}} C \bar{\sigma}^{k_{j}}$ and $\bar{\sigma}_{1}^{l_{1}} \cap \bar{\sigma}_{2}^{l_{2}} \neq \emptyset$, in fact $\sigma_{1}^{l_{1}} \cap \sigma_{2}^{l_{2}}=C^{l_{1}+l_{2}-n} \neq \emptyset$ and

$$
\left|C^{l_{1}+l_{2}-n}\right| \geqq c(k, \Theta, \delta) \eta_{1}^{l_{1}+l_{2}-n} .
$$

(2) For all nonempty $C^{l_{1}+l_{2}-n}$ as above, there exists $p \in C^{l_{1}+l_{2}-n}$ with

$$
\operatorname{dist}\left(p, \partial \bar{C}^{l_{1}+l_{2}-n}\right)>c(k, \Theta, \delta) \cdot \eta_{1} .
$$

(3) If $C^{\prime}$ is formed using the points $p$ of (7.3), then each simplex $\psi^{m}$ of $C^{\prime}$ has a fatness which satisfies

$$
\Theta\left(\psi^{m}\right) \geqq\left|\psi^{m}\right| / \eta_{1}^{m} \geqq c(k, \Theta, \delta)
$$

Proof. We can assume that $\bar{\sigma}_{1}^{k_{1}}$ has one vertex at the origin. Let $\sigma_{j}^{k_{j}}(j=1,2)$ be any simplices as above with $\sigma_{1}^{k_{1}} \pitchfork_{\delta} \sigma_{2}^{k_{2}}$. We begin by establishing the following:

Claim. The set $\left\{\sigma_{1}^{k_{1}} \cap \sigma_{2}^{k_{2}}\right\}$ of all possible intersections is unchanged, if we add the (possibly empty) condition that $\sigma_{2}^{k_{2}}$ has mesh at most $c(\Theta)(1+\delta) \eta_{1}$ for a suitable $c(\Theta)$.

Proof of Claim. In fact, for each $\sigma_{2}^{k_{2}}$ we can find a simplex $\omega_{2}^{k_{2}}$ which is similar to $\sigma_{2}^{k_{2}}$, such that $\sigma_{1}^{k_{1}} \pitchfork_{\delta} \omega_{2}^{k_{2}}, \sigma_{1}^{k_{1}} \cap \omega_{2}^{k_{2}}=\sigma_{1}^{k_{1}} \cap \sigma_{2}^{k_{2}}$ and for each $\left(k_{2}-1\right)$-face $\omega_{2}^{k_{2}-1} \subset \bar{\omega}_{2}^{k_{2}}$, we have $\operatorname{dist}\left(\bar{\sigma}_{1}^{k_{1}}, \bar{\omega}_{2}^{k_{2}-1}\right) \leqq \delta \eta_{1}$ (see Fig. 7.1). 
Fig. 7.1
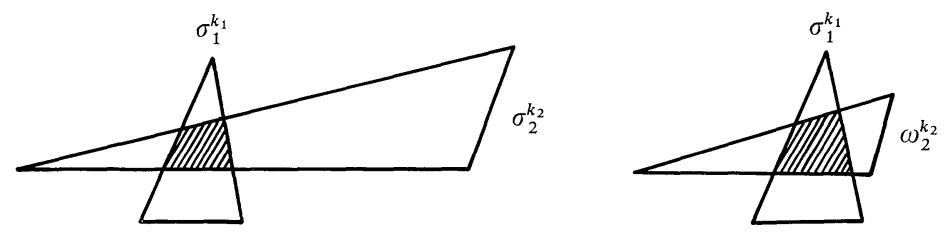

It follows there is a point $g \in \omega_{2}^{k_{2}}$ such that its distance to all $k_{2}-1$-faces of $\bar{\omega}_{2}^{k_{2}}$ is $\leqq(1+\delta) \eta_{1}$. Consider the compact set of simplices $\sigma^{k_{2}}$ of fatness $\leqq \Theta$, mesh 1 and a fixed vertex. The positive continuous function

$$
f\left(\sigma^{k_{2}}\right)=\inf _{g \in \sigma^{k_{2}}} \sup _{\sigma^{k_{2}-1} \subset \sigma^{k_{2}}} \operatorname{dist}\left(g, \sigma^{k_{2}-1}\right)
$$

attains a positive minimum $\frac{1}{c(\Theta)}$ and $c(\Theta)$ satisfies the claim.

Now consider the compact space $S(\Theta, \delta)$ of all pairs $\sigma_{1}^{k_{1}}, \sigma_{2}^{k_{2}}$ such that

๙) $\sigma_{1}^{k_{1}}$ has a fixed vertex at $0 \in E^{k}$,

ק) $\sigma_{1}^{k_{1}}$ and $\sigma_{2}^{k_{2}}$ have fatness $\geqq \Theta$, and mesh $1,2(1+\delta)$, respectively.

ү) $\bar{\sigma}_{1}^{k_{1}} \cap \bar{\sigma}_{2}^{k_{2}} \neq \emptyset$.

We are now ready to establish 1)-3) of Lemma 7.1.:

1) Since $S(\Theta, \delta)$ is compact, it suffices to show $\bar{\sigma}_{1}^{l_{1}} \cap \bar{\sigma}_{2}^{l_{2}} \neq 0$ implies $\sigma_{1}^{l_{1}} \cap \sigma_{2}^{l_{2}} \neq \emptyset$. For then $\left|C^{l_{1}+l_{2}-\eta}\right|$ is a positive continuous function on a compact subset, and hence attains a positive minimum.

But if $\bar{\sigma}_{1}^{l_{1}} \cap \bar{\sigma}_{2}^{l_{2}} \neq 0$, then for some $\sigma_{j}^{m_{j}} \subset \bar{\sigma}_{j}^{l_{j}}$ we have $\sigma_{1}^{m_{1}} \cap \sigma_{2}^{m_{2}} \neq \emptyset$. Let $m_{j}$ be maximal with respect to this property. Assume $m_{1}<l_{1}$ and pick $g \in \sigma_{1}^{m_{1}} \cap \sigma_{2}^{m_{2}}$. Near $g, \sigma_{1}^{l_{1}}$ has a product neighborhood $\sigma_{1}^{m_{1}} \times C_{0, \varepsilon}\left(L^{l_{1}-m_{1}-1}\right)$, where $L^{l_{1}-m_{1}-1}$ is the link of $\sigma_{1}^{m_{1}}$ in $\sigma_{1}^{l_{1}}$. Now since $\sigma_{1}^{m_{1}} \pitchfork_{\delta} \sigma_{2}^{m_{2}}$ and $\sigma_{1}^{m_{1}} \cap \sigma_{2}^{m_{2}} \neq \emptyset$, we must have $m_{1}+m_{2} \geqq n$. But then the transversality condition on the planes $\left[\sigma_{1}^{m_{1}}\right]$ and $\left[\sigma_{2}^{m_{2}}\right]$ implies that the orthogonal projection of $\sigma_{2}^{m_{2}}$ onto the subspace $\mathscr{E}^{n-m_{1}}$ through $g$ and orthogonal to $\mathscr{E}_{1}^{m_{1}}$ is an open set of $\mathscr{E}^{n-m_{1}}$ containing $g$. But this easily implies that $\sigma_{1}^{m_{1}} \times C_{0, \varepsilon}\left(L^{l_{1}-m_{1}-1}\right) \cap \sigma_{2}^{m_{2}} \neq \emptyset$ and thus $\sigma_{1}^{l_{1}} \cap \sigma_{2}^{m_{2}} \neq \emptyset$. This contradicts the maximality of $m_{1}$. Therefore $m_{1}=l_{2}$ and similarly $m_{2}=l_{2}$.

2) Clearly each $m$-cell $\bar{C}^{m}$ satisfies

$$
\left|C_{\alpha}^{m}\right| \leqq \eta_{1}^{m}
$$

Moreover, the number of faces $\bar{\sigma}_{1}^{l_{1}} \cap \bar{\sigma}_{2}^{l_{2}}$ of $\bar{C}^{m}$ is $\leqq 2^{k_{1}+k_{2}+2}$. Let $p \in C^{m}$ be at maximal distance $\varrho$ from $\partial \bar{C}^{m}$. Then if $T_{\varrho}\left(C^{t}\right)$ denotes the set of points of distance $\leqq \varrho$ from $C^{t} \subset \partial \bar{C}^{m}$, we have

Clearly

$$
\bar{C}^{m} \subset \bigcup_{C^{t} \subset \partial \bar{C}^{m}} T_{\varrho}\left(C^{t}\right) .
$$

$$
\left|T_{\varrho}\left(C^{t}\right) \cap C^{m}\right| \leqq c_{m} \varrho^{m-t}\left|C^{t}\right| .
$$

Combining (7.6)-(7.8) give

$$
c_{1}(k, \Theta, \delta) \eta_{1}^{m} \leqq \sum_{t=0}^{m-1} \varrho^{m-t} \eta_{1}^{t},
$$

from which (7.3) follows. 
3) Relation (7.4) follows from (7.2) and (7.3) by induction.

To proceed further, we make note of another compactness property.

Lemma 7.2. Fix $\Theta, \eta$ and $r>0$. Let $K^{n} C E^{n}$ be a euclidean simplicial complex of fatness $\geqq \Theta$ and mesh $\eta$ such that every point of $K^{n}$ is contained in a closed $n$-simplex. Then the number \#(r) of simplices of $K^{n}$ which are contained in a ball $B_{r}(p)$ satisfies

$$
\begin{aligned}
\#(r) & \leqq 2^{n+1}\left|B_{r}(p)\right| / \Theta \cdot \eta^{n} \\
& \leqq c(n) \Theta^{-1}\left(\frac{r}{\eta}\right)^{n} .
\end{aligned}
$$

In particular, if we consider all such complexes containing a fixed vertex $p$, then the set $\{s t(p)\}$ of all stars of $p$, forms a compact space in a natural way. Thus the number of distinct combinatorial structures on $\{s t(p)\}$ depends only on $\Theta$.

Proof. Relation (7.10) is immediate from the definition of $\Theta$. The compactness of $\{s t(p)\}$ follows since each configuration is determined by at most $\#\left(\sigma^{1}\right) \leqq c_{n} \Theta^{-1}$ edge lengths, which vary in the interval $[\eta \Theta, \eta]$.

The basic induction step of our argument to prove Lemma 6.3 can now be described. Let $K_{1}^{n}, K_{2}^{n} \subset E^{n}$. Assume that every point of $K_{j}^{n}$ is contained in a closed $n$-simplex. Let $K_{1}^{n}$ and $K_{2}^{n}$ have fatness $\geqq \Theta$ and mesh $\eta_{1} \leqq \eta_{2}$ respectively. Fix $\sigma_{1}^{0} \in K_{1}^{n}$ and $\hat{\sigma}_{1}^{0} \in E^{n}$. Let $\hat{K}_{1}^{n}$ denote the complex obtained from $K_{1}^{n}$ by moving $\sigma_{1}^{0}$ to $\hat{\sigma}_{1}^{0}$, while keeping the other vertices of $K_{1}^{n}$ fixed. Let $L_{2} \subset K_{2}^{n}$ consists of those simplices $\sigma_{2}^{l}$ such that $\sigma_{2}^{l} \cap B_{2 \eta_{1}}\left(\sigma_{1}^{0}\right) \neq 0$.

Lemma 7.3. For all $\Theta, \psi, \varepsilon>0$ there exists $\delta(\Theta, \psi, \varepsilon)>0$ with the following property. Let $K_{1}^{n}, K_{2}^{n}$ be as above. Assume that for some $i \geqq 0$ all $\sigma_{1}^{i-1} C \partial \operatorname{st}\left(\sigma_{1}^{0}\right)$ satisfy

$$
\sigma_{1}^{i-1} \pitchfork_{\psi} L_{2} \text {. }
$$

Then there exists $\hat{\sigma}_{1}^{0}$, with $\operatorname{dist}\left(\sigma_{1}^{0}, \hat{\sigma}_{1}^{0}\right)<\varepsilon \cdot \eta_{1}$ such that for all $\hat{\sigma}^{i} \subset \operatorname{st}\left(\hat{\sigma}_{1}^{0}\right) \backslash \partial \operatorname{st}\left(\hat{\sigma}_{1}^{0}\right)$

$$
\hat{\sigma}_{1}^{i} \pitchfork_{\delta(\Theta, \psi, \varepsilon)} L_{2} \text {. }
$$

Since (7.11) and (7.12) actually involve conditions on the subspaces $\left[\sigma^{l}\right]$ generated by the simplexes $\sigma^{l}$, to prove Lemma 7.3, it will be convenient to begin by considering arbitrary subspaces $E^{l_{1}-1} \subset E^{l_{1}}, E^{l_{2}}$ through $0 \in E^{n}$. Set $E^{l_{1}} \cap E^{l_{2}}=G^{t}$.

Lemma 7.4. If $E^{l_{1}-1} \pitchfork E^{l_{2}}$, either $E^{l_{1}} \pitchfork E^{l_{2}}$ or $l_{1}+l_{2}<n, \quad t=1$ and $\operatorname{dim}\left(E^{l_{1}-1} \cap G^{1}\right)=0$. In the latter case, let $\|g\|=1,[g]=G^{1}$ and choose $h \in\left[E^{l_{1}} \cup E^{l_{2}}\right]^{\perp},\|h\|=1$. Setting $E_{\varepsilon}^{l_{1}}=\left[g+\varepsilon h \cup E^{l_{1}-1}\right]$ we have $E_{\varepsilon}^{l_{1}} \pitchfork E^{l_{2}}$ whenever $\varepsilon>0$.

Proof. Either $E^{l_{1}} \pitchfork E^{l_{2}}$ or $t \geqq 1$ and $l_{1}+l_{2}-n<t$. If $t>1$, then

$$
\begin{aligned}
0<t-1 & =\operatorname{dim}\left(E^{l_{1}-1} \cap G^{t}\right) \\
& =\operatorname{dim}\left(E^{l_{1}-1} \cap E^{l_{2}}\right) \\
& =\left(l_{1}-1\right)+l_{2}-n,
\end{aligned}
$$

where the last equality is implied by the transversality, $E^{l_{1}-1} \pitchfork E^{l_{2}}$, of $E^{l_{1}-1}$ and $E^{l_{2}}$. Since (7.13) contradicts $l_{1}+l_{2}-n<t$, in fact $t=1$. The rest is obvious. 
Now let $E^{l_{1}-1} \subset E^{l_{1}}, E^{l_{2}}$ be as above with $E^{l_{1}-1} \pitchfork E^{l_{2}}, E^{l_{1}} \pitchfork E^{l_{2}}$. Let $\mathscr{E}^{l_{1}-1} \subset \mathscr{E}^{l_{1}}, \mathscr{E}^{l_{2}}$ be parallel affine subspaces. Assume that $l_{1}+l_{2}<n$ and that $\mathscr{E}^{l_{1}-1} \cap \mathscr{E}^{l_{2}}=\emptyset$. Then $\mathscr{E}^{l_{1}} \cap \mathscr{E}^{l_{2}}$ is either empty or a single point $w$. In the latter case let $v \in \mathscr{E}^{l_{1}-1}$ be closest to $w$. Let $h \in\left[E^{l_{1}} \cup E^{l_{2}}\right]^{\perp},\|h\|=1$. Set

$$
E_{\varepsilon}^{l_{1}}=\left[\frac{w-v}{\|w-v\|}+\varepsilon h \cup E^{l_{1}-1}\right] \quad \text { and } \quad \mathscr{E}_{\varepsilon}^{l_{1}}=v+E_{\varepsilon}^{l_{1}}
$$

(see Fig. 7.2 with $l_{1}=l_{2}=1, n=3$ ). The following lemma is obvious.

Lemma 7.5. For $\varepsilon>0, \mathscr{E}_{\varepsilon}^{l_{1}} \cap \mathscr{E}^{l_{2}}=\emptyset$.

Fig. 7.2

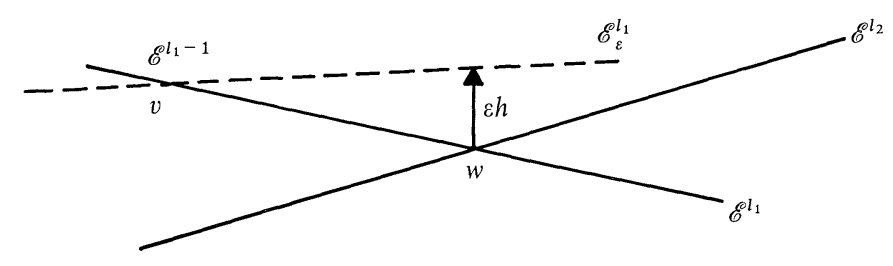

Proof of Lemma 7.3. Let $K_{1}^{n}, K_{2}^{n}, \sigma_{1}^{0}, \Theta, \psi$ and $\varepsilon$ be given. It will suffice to show that there exists $\hat{\sigma}_{1}^{0}, \delta>0$ as in (7.12), where $\delta$ might depend on $K_{1}^{n}, K_{2}^{n}$, and $\sigma_{1}^{0}$. Then a straightforward compactness argument based on Lemma 7.2 shows that $\delta$ can be chosen to depend only $\Theta, \psi$, and $\varepsilon$.

Choose orderings $\sigma_{2,1}<\sigma_{2,2}, \ldots, \sigma_{2, m_{2}}$ of all simplexes of $L_{2}$ and $\sigma_{1,1}^{i-1}<\sigma_{1,2}^{i-1}$ $<\ldots<\sigma_{1, m_{1}}^{i-1}$ of the $(i-1)$-simplexes of $\partial \operatorname{st}\left(\sigma_{1}^{0}\right)$. Each $i$-simplex $\sigma_{1, \alpha}^{i} C \operatorname{st}\left(\sigma_{1}^{0}\right) \backslash \partial \operatorname{st}\left(\sigma_{1}^{0}\right)$ is the convex hull $\left\{\sigma_{1}^{0}, \sigma_{1}^{i-1}\right\}$ of $\sigma_{1}^{0}$ and some unique $\sigma_{1, \alpha}^{i-1} \in \partial \operatorname{st}\left(\sigma_{1}^{0}\right)$. Let

$$
\mathscr{E}_{1}^{i-1}=\left[\sigma_{1,1}^{i-1}\right] \subset \mathscr{E}_{1}^{i}=\left[\sigma_{1,1}^{i}\right] \text { and } \mathscr{E}_{2}^{l_{2}}=\left[\sigma_{2,1}^{l_{2}}\right]
$$

Let $\mathscr{E}_{1, \varepsilon_{1,1}}^{i}$ be obtained from $\mathscr{E}_{1}^{i}$ by applying Lemma 7.4 and (if necessary) Lemma 7.5 successively with $\varepsilon_{1,1}$ small. Let $p_{1,1}$ be the point in $\mathscr{E}_{1, \varepsilon_{1,1}}^{i}$ closest to $\sigma_{1}^{0}$. Then $\left[p_{1,1} \cup \sigma_{1,1}^{i-1}\right] \uparrow \sigma_{2,1}$. Repeat the argument with $\sigma_{2,1}$ replaced by $\sigma_{2,2}$ and $\sigma_{1}^{0}$ replaced by $p_{1,1}$ to obtain $p_{1,2}$. If $\varepsilon_{1,2}$ is chosen sufficiently small, we will have both $\left[p_{1,2} \cup \sigma_{1,1}^{i-1}\right] \pitchfork \sigma_{2,1}$ and $\left[p_{1,2} \cup \sigma_{1,1}^{i-1}\right] \pitchfork \sigma_{2,2}$. Proceeding in this fashion, we obtain $p_{1, m_{2}}$ with $\left[p_{1, m_{2}} \cup \sigma_{1,1}^{i-1}\right] \pitchfork L_{2}$. Repeating the argument with $\sigma_{1,2}^{i-1}, \ldots, \sigma_{1, m_{1}}^{i-1}$, (at each stage choosing $\varepsilon_{\alpha, \beta}$ sufficiently small so as not to destroy the transversality already achieved), we obtain $\hat{\sigma}_{1}^{0}=p_{m_{1}, m_{2}}$ with $\left[\hat{\sigma}^{0} \cup \sigma_{\alpha}^{i-1}\right] \pitchfork L^{2}$, for all $\alpha$.

We can now give the proof of Lemma 6.3.

Proof of Lemma 6.3. An obvious compactness argument shows that for all $\Theta>0$, there exists $f(\Theta)>0$ with the following property. If $\sigma^{k} \subset E^{n}$ has fatness $\Theta$ and mesh $\eta_{1}$, and each vertex of $\sigma^{k}$ is moved by at most $f(\Theta) \eta_{1}$, the resulting simplex $\omega^{k}$ has fatness $\geqq \Theta / 2$.

Similarly, (using Lemma 7.2), given $\Theta, \psi>0$, there exists $g(\Theta, \psi)$ with the following property. Let $\sigma^{k}$ be as above and let $K^{n} \subset E^{n}$ have fatness $\Theta$ and mesh $\eta_{2} \geqq \eta_{1}$. Assume that every point of $K^{n}$ is contained in a closed $n$-simplex and that $\sigma^{k} \pitchfork_{\psi} K^{n}$. Then if each vertex of $\sigma^{k}$ is moved by at most $g(\Theta, \psi) \eta_{1}$, the resulting simplex $\omega^{k}$ satisfies $\omega^{k} \pitchfork_{\psi / 2} K^{n}$. 
Now, as in Lemma 6.3, let $K_{j}^{n}$ be complexes such that every point of $B_{r}(0) \subset E^{n}$ is contained in an $n$-simplex of $K_{j}^{n}$ and every $n$-simplex of $K_{j}^{n}$ intersects $B_{r}(0)$. Let $K_{j}^{n}$ have fatness $\Theta$ and mesh $\eta_{j}$, with $\eta_{1} \leqq \eta_{2}$. Define subcomplexes of $K_{1}^{n}, K_{2}^{n}$ as follows.

$$
\begin{aligned}
L_{2}^{n} & =\left\{\bar{\sigma}^{l} \subset K_{2}^{n} \mid \bar{\sigma}^{l} \subset B_{r}(0), \eta_{1} \leqq \operatorname{dist}\left(\bar{\sigma}^{l}, \partial B_{r}(0)\right) \leqq \eta_{2}\right\}, \\
M_{2}^{n} & =\left\{\sigma^{l} \subset K_{2}^{n} \mid \bar{\sigma}^{l} \subset \bar{\sigma}^{n} \subset B_{r}(0), \bar{\sigma}^{n} \cap L_{2}^{n} \neq \emptyset\right\}, \\
P_{1}^{n} & =\left\{\bar{\sigma}^{l} \subset K_{1}^{n} \mid \operatorname{dist}\left(\bar{\sigma}^{l}, L_{2}^{n}\right) \leqq \eta_{1}\right\}, \\
Q_{1}^{n} & =\left\{\sigma^{l} \subset K_{1}^{n} \mid \bar{\sigma}^{l} \subset \sigma^{n} \subset B_{r}(0), \bar{\sigma}^{n} \cap P_{1}^{n} \neq \emptyset\right\} .
\end{aligned}
$$

Fig. 7.3

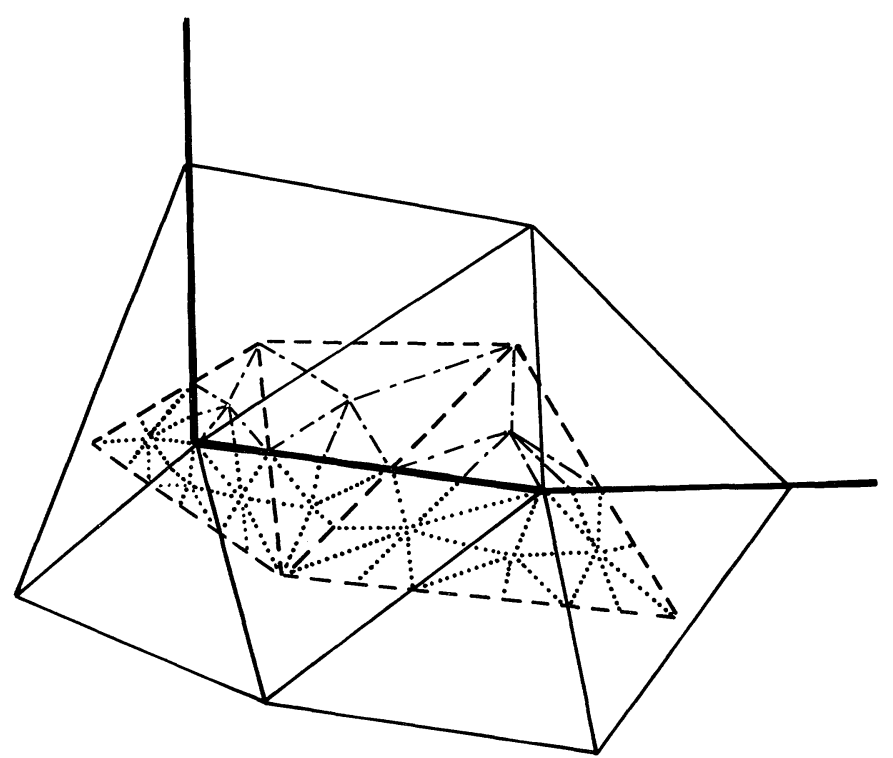

Let $\Sigma^{i}(T)$ denote the $i$-skeleton of the complex $T$.

Choose an ordering of the vertices of $P_{1}^{n}$. We are going to move each vertex in order a distance at most $d_{i}, i=1, \ldots, n$, to obtain complexes $K_{1}^{n}, K_{1,0}^{n}, \ldots, K_{1, n-1}^{n}$ which will agree with $K_{1}^{n}$ outside of $Q_{1}^{n}$. When all moves are complete $P_{1, n-1}^{n} C$ $K_{1, n-1}^{n}$ will be in general position relative to $K_{2}^{n}$ by a definite amount. Set

$$
d_{0}=\eta_{1} \cdot \frac{1}{n} \min \left(\frac{1}{2}, f(\Theta)\right)
$$

By Lemma 7.3, if we move the vertices of $P_{1}^{n}$ by at most $d_{0}$, we can insure that

$$
\Sigma^{0}\left(P_{1,0}^{n}\right) \pitchfork_{\psi_{0}} K_{2}^{n}
$$

for some

$$
\psi_{0}=\delta\left(\Theta, 1, \frac{d_{0}}{\eta_{1}}\right)
$$


For $i=1, \ldots, n-1$, we inductively define

$$
\begin{gathered}
d_{i}=\eta_{1} \cdot \frac{1}{n} \min \left(\frac{n}{2}, f(\Theta), g\left(\frac{\Theta}{2}, \frac{\psi_{0}}{2}\right), \ldots, g\left(\frac{\Theta}{2}, \frac{\psi_{i-1}}{2}\right)\right), \\
\psi_{i}=\delta\left(\frac{\Theta}{2}, \psi_{i-1}, \frac{d_{i}}{\eta_{1}}\right) .
\end{gathered}
$$

Then $d_{0} \geqq d_{1} \geqq \ldots \geqq d_{n-1}$ and by (7.14) and the definition of $\frac{f(\Theta)}{n} \eta_{1}$, the fatness of $K_{1, i}^{n}$ is at least $\frac{\Theta}{2}$, for all $i$. It follows by Lemma 7.3 and induction that we can insure that

and for $j>i$

$$
\Sigma^{i}\left(P_{1, i}^{n}\right) \pitchfork_{\psi_{\imath}} K_{2}^{n}
$$

$$
\Sigma^{i}\left(P_{1, j}^{n}\right) \pitchfork_{\psi_{2} / 2} K_{2}^{n}
$$

Thus,

$$
P_{1, n-1}^{n} \pitchfork_{\psi} K_{2}^{n}
$$

with

$$
\psi=\frac{1}{2} \min \left(\psi_{0}, \ldots, \psi_{n-1}\right) .
$$

Now use Lemma 7.1 to form the barycentric subdivision of $P_{1, n-1}^{n} \cap L_{2}^{n}$. Extend $\left(P_{1, n-1}^{n} \cap L_{2}^{n}\right)^{\prime}$ to a subdivision of $M_{2}^{n}$ as follows. Let $p^{i}$ denote either the barycenter of some $\sigma^{i} \in M_{2}^{n} \backslash L_{2}^{n}$ with $\bar{\sigma}^{i} \cap L_{2}^{n} \neq \emptyset$, or a vertex of $\left(P_{1, n-1}^{n} \cap L_{2}^{n}\right)^{\prime}$ lying in some $\bar{\sigma}^{i} \subset \partial L_{2}^{n} \cap M_{2}^{n}$. Subdivide each $\sigma^{n} \subset M_{2}^{n} \backslash L_{2}^{n}$ by forming all simplices with vertices $p^{i_{1}}, \ldots, p^{i_{j}}$, where $i_{1}<\ldots<i_{j}$. The resulting triangulation $\tilde{K}_{2}^{n}$ extends $\overline{K_{2}^{n} \backslash M_{2}^{n}}$; (see Fig. 7.3). Similarly, using $Q_{1, n-1}^{n}$ we construct $\tilde{K}_{1}^{n}$ which extends $\overline{K_{1}^{n} \backslash Q_{1, n-1}^{n}}$ and agrees with $\left(P_{1, n-1}^{n} \cap L_{2}^{n}\right)^{\prime}$ near $\partial \tilde{K}_{1}^{n}$. This completes the proof.

\section{Convergence of Boundary Curvatures}

Let $M^{n}$ be a Riemannian manifold with boundary. As described at the end of Sect. 1 we can consider smooth triangulations of $M^{n}$ and form corresponding piecewise flat approximations $M_{\eta}^{n}$. It is convenient to assume that $M^{n}$ is given as a subset of the interior of larger Riemannian manifold $V^{n}$. Thus we have ambient geodesics between closeby points of $\partial M^{n}$, even if $\partial M^{n}$ is not convex. All curvature bounds we assume will refer to $V^{n}$.

Now recall from Sect. 3 the definition of the total exterior $j^{\text {th }}$ Lipschitz-Killing curvatures $H^{j}=H^{j}\left(M^{n}\right)$. It is the contribution to (3.23), (3.38), or (3.39) arising from those $\sigma^{n-j}$ simplices which belong to $\partial M_{\eta}^{n}$. In analogy to what was done for $R^{j}$ and $R_{\eta}^{j}$ we now define measures

$$
U \rightarrow H^{j}(U), \quad U \rightarrow H_{\eta}^{j}(U)
$$

where $U$ is a bounded open (bounded) set of $\partial M^{n}$. Again, we will assume that the volume of $T_{r}(U)$ in $\partial M^{n}$, the set of points in $\partial M^{n}$ with distance $\leqq r$ to the boundary of $U$ in $\partial M^{n}$, goes to zero with $r$. The second fundamental form of $\partial M^{n}$ will be denoted by $I I$. 
Theorem 8.1. There are constants $c=c(\|R\|,\|\nabla R\|,\|I I\|,\|\nabla I I\|, \Theta)$ and $\eta_{0}=\eta_{0}(\|R\|,\|\nabla R\|,\|I I\|,\|\nabla I I\|, \Theta)>0$ such that

$$
\left|H_{\eta}^{j}(U)-H^{j}(U)\right| \leqq c\left(|U| \sqrt{\eta}+\left|T_{\sqrt{\eta}}(\partial U)\right|\right)
$$

whenever $\eta \leqq \eta_{0}$ and $M_{\eta}^{n}$ has fatness $\geqq \Theta$. In particular as $\eta$ tends to zero, $H_{\eta}^{j}(U)$ converges to $H^{j}(U)$ whenever $\left\{M_{\eta}^{n}\right\}$ is such that $\eta \rightarrow 0$ with $\Theta(\eta) \geqq \Theta_{0}>0$.

By our previous results on interior Lipschitz-Killing curvatures and the remark following (3.39), it suffices to restrict attention to the case where $j$ is even. The idea of the proof is the same as for the interior curvatures, but now we use the extension of Gilkey's characterization of the Lipschitz-Killing curvatures to the boundary terms. The polynomial $* H^{j}(p)[$ see $(0.4)]$ is characterized by the following properties.

1) It is invariant under the orthogonal subgroup of $O\left(M_{p}^{n}\right)$, which leaves the normal direction invariant.

2) It vanishes if the manifold $M^{n}$ splits locally and metrically as $S^{1} \times \ldots \times S^{1} \times M^{n-j-1}\left(j+1\right.$ factors $\left.S^{1}\right)$.

3) It agrees with $* H^{j}(p)$ for the family of curvature tensors obtained from the spaces $S^{1} \times \ldots \times S^{1} \times S^{2} \times \ldots \times S^{2} \times D^{j-2 k}\left[n-j\right.$ factors $S^{1}, k$ factors $S^{2}(2 k \leqq j)$, $D^{j-2 k}=$ unit disc in $\left.E^{j-2 k}\right]$.

The above characterization follows immediately by combining Theorem $2.2 \mathrm{~b}$ of [G2] with the argument of [D], in which the interior curvatures $R^{j}$ are characterized as in Sect. 1.

As in Sect. 5, we begin by considering a metric ball $\underline{B}_{r}(p) \subset \partial M$ and proving the existence of a polynomial $\mathscr{H}^{j}(r, \tilde{T})$ in $R, I I$, such that the following lemma holds (the lift $\tilde{T}$ is defined below).

Lemma 8.2. There is a polynomial $\mathscr{H}^{j}(r, \tilde{T})$ in $R, I I$ such that

$$
\left|\mathscr{H}^{j}(r, \tilde{T})-\frac{1}{\left|\underline{B}_{r}(p)\right|} H_{\eta}^{j}\left(\underline{B}_{r}(p)\right)\right| \leqq c r,
$$

where $c=c(\|R\|,\|\nabla R\|,\|I I\|,\|\nabla I I\|, \Theta)$ and

$$
\left|\mathscr{H}^{j}(r, \tilde{T})\right| \leqq c(\|R\|,\|I I\|, \Theta) .
$$

This yields

Lemma 8.3. For any $U \subset \partial M^{n}$ there is a uniform bound of the form

$$
\left|\mathscr{H}_{\eta}^{j}(U)\right| \leqq c\left(\|R\|+\|I I\|^{2}\right)^{j / 2}\left|T_{\eta}(U)\right|
$$

where $c$ is as in (8.2).

Now, the application of a straightforward extension of Lemma 6.3 to the boundary case, yields the following analog of Lemma 5.4

Lemma 8.4. There is a constant $c$ as in (8.2) such that if the triangulations $T_{1}, T_{2}$ with mesh $\eta_{1}, \eta_{2}$ have fatness $\geqq \Theta>0$, then

$$
\left|\mathscr{H}^{j}\left(r, \tilde{T}_{1}\right)-\mathscr{H}^{j}\left(r, \tilde{T}_{2}\right)\right| \leqq c\left(\frac{\eta_{1}+\eta_{2}}{r}+r\right) .
$$


If we assume the above lemmas, the proof of Theorem 8.1 can be easily completed.

Proof of Theorem 8.1. The verification of 1) and 2) of Gilkey's axioms is completely analogous to the argument for the interior case. To check the normalization, as in the interior case, we are reduced to $S^{2} \times \ldots \times S^{2} \times D^{j-2 k}$ ( $k$ factors $S^{2}$ ) and the boundary term for the Chern-Gauss-Bonnet form. However, since the interior of $D^{j-2 k}$ is flat, the interior terms vanish in both the smooth and piecewise flat case. Thus the equivalence of the boundary terms follows from Chern-Gauss-Bonnet theorem in the piecewise flat case, together with the explicit evaluation of the boundary term for $\partial D^{j-2 k}$ in the smooth case. This completes the proof.

Since Lemma 8.3 is an immediate consequence of Lemma 8.2, it will suffice to verify Lemmas 8.2 and 8.4 .

First, we begin the construction of the polynomial $\mathscr{H}^{j}(r, \tilde{T})$. Let $\exp _{p} x$ be the exponential map of $\partial M^{n}$. Let $M_{p}^{+} \subset M_{p}$ denote the half space $\{(x, t N)\}$, where $x$ is tangent to $\partial M^{n}, N$ is the inward unit normal, and $t>0$. Define a local diffeomorphism $\phi: M_{p}^{+} \rightarrow M$ by

$$
\phi(x, t N)=\exp _{\underline{\exp _{p}} x} t N .
$$

Using $\phi$, we can define the lift $\tilde{T}$, of $T$ to $M_{p}^{+}$.

The construction starts with the evaluation of the pointwise limit of $P_{\chi}\left(C^{\perp}\left(\sigma^{n-j}\right)\right)$ at a vertex $p_{1}$ of $\sigma^{n-j} C \partial M^{n}$. Here a new point will arise. To calculate $P_{\chi}^{\chi}\left(\sigma^{n-j}\right)$ we compare with the decomposition normal cone to $0 \in M_{p}^{+}$induced by the triangulation $\tilde{T}$. As before, we view all dihedral angles as functions of the $(0,2)$ angles, but now we must take into account the fact that there are three distinct types of $(0,2)$ 's.

Let $p_{1}, p_{2}, p_{3}$ be the vertices of $\sigma^{2} \in T$, with $p_{1} \in \partial M$. Let $\alpha_{j}$ be the corresponding angles, and $\alpha_{j}^{e}$ the angles of the Euclidean triangle with the same edge lengths. Then if $p_{2}, p_{3} \in \operatorname{int}(M)$, as in Sect. 4 ,

$$
\begin{aligned}
\alpha_{1}-\alpha_{1}^{e} & =\frac{1}{3} K\left|\sigma^{2}\right|+O\left(\eta^{3}\right) \\
& =O\left(\eta^{2}\right) .
\end{aligned}
$$

Similarly if $p_{2}, p_{3} \in \partial M$,

$$
\begin{aligned}
\alpha_{1}-\alpha_{1}^{e} & =\frac{1}{3} \hat{K}\left|\sigma^{2}\right|+O\left(\eta^{3}\right) \\
& =O\left(\eta^{2}\right),
\end{aligned}
$$

where $\hat{K}$ is the curvature of the plane section determined by $\sigma^{2}$, in the metric induced on the boundary.

Now suppose that say $p_{2} \in \partial M$ and $p_{3} \in \operatorname{int}(M)$. Let $\gamma_{1}, \gamma_{2}, \gamma_{3}$ denote the edges of $\sigma^{2}$ where $\gamma_{1}, \gamma_{2}$ are interior geodesics and $\gamma_{3}$ is a boundary geodesic. Let $\gamma_{3}$ denote the interior geodesic from $p_{1}$ to $p_{2}$. Finally let $\beta_{j}$ denote the angles of the triangle with sides $\gamma_{j}$ and $\beta_{j}^{e}$ the angles of the Euclidean triangle with the same edge lengths. Then

$$
\alpha_{1}-\alpha_{1}^{e}=\left(\alpha_{1}-\beta_{1}\right)+\left(\beta_{1}-\beta_{1}^{e}\right)+\left(\beta_{1}^{e}-\alpha_{1}^{e}\right) .
$$


A standard calculation in normal coordinates shows that

$$
\begin{aligned}
\alpha_{1}-\beta_{1} & =\frac{1}{2} I I\left(\underline{\gamma}_{3}^{\prime}, \underline{y}_{3}^{\prime}\right) \cdot \underline{l}_{3}+c \cdot \eta^{3} \\
& =O(\eta),
\end{aligned}
$$

where $c=c(\|R\|,\|\nabla R\|,\|I I\|,\|\nabla I I\|, \Theta)$ and $\underline{l}_{3}$ is the length of $\underline{\gamma}_{3}$. As in Sect. 4

$$
\left(\beta_{1}-\beta_{1}^{e}\right)=\frac{1}{3} K\left|\sigma^{2}\right|+c \eta^{3},
$$

and by another standard calculation

$$
\left(\beta_{e}^{1}-\alpha_{1}\right)=-\frac{1}{96} I I\left(\underline{\gamma}_{3}^{\prime}, \underline{\gamma}_{3}^{\prime}\right)^{2} \frac{\left(l_{2}^{2}-l_{1}^{2}-l_{3}^{2}\right)}{\left|\sigma^{2}\right|} l_{3}^{2},
$$

where $l_{j}$ is the length of $\gamma_{j}$. Thus,

$$
\alpha_{1}-\alpha_{1}^{e}=O(\eta)
$$

Now suppose we attempt to calculate the limiting value of $P_{\chi}\left(C^{\perp}\left(\sigma^{n-j}\right)\right)$ by using (3.49) as in Proposition 3.2. Since the right hand side is only $O(\eta)$ and not $O\left(\eta^{2}\right)$, it is not immediately obvious that we obtain a polynomial of degree $j$ in $\eta$, when $\sigma^{n-j} C \sigma^{n}$ and $\sigma^{n}$ contains several interior vertices.

Proof of Lemma 8.2. Let $\sigma^{n-j} C \partial M$, and let $\sigma^{0}$ be a vertex of $\sigma^{n-j}$. We define a 1-parameter family of metrics $g_{t}$ near $\sigma^{0}$ as in (6.16). By means of the corresponding 1-parameter family of piecewise flat approximations $M_{\eta, t}$ we can connect $M_{\eta}=M_{\eta, 1}$ to the flat configuration on $M_{\sigma^{0}}^{+}$. As in (3.44) we write

$$
P_{\chi}\left(C^{\perp}\left(\sigma^{n-j}\right)\right)=-\int_{0}^{1} \sum\left(\sigma^{n-j}, \sigma^{n-j+2}\right)^{\prime} P_{\chi}\left(C^{\perp}\left(\sigma^{n-j+2}\right)\right) d t .
$$

Since the case $j=n-2$ follows from (8.7), (8.8), (8.13) as in the interior case, we can assume by induction that

$$
P_{\chi}\left(C^{\perp}\left(\sigma^{n-j+2}\right)\right)=O\left(\eta^{j-3}\right)
$$

In view of (8.9), this implies

$$
\left(\sigma_{t}^{n-j}, \sigma_{t}^{n-j+2}\right)^{\prime} P_{\chi}\left(C^{\perp}\left(\sigma_{t}^{n-j+2}\right)\right)=O\left(\eta^{j-1}\right),
$$

provided $\sigma^{n-j+2} C \partial M^{n}$. But if $\sigma^{n-j+2} \not \subset \partial M$, then $P_{\chi}\left(C^{\perp}\left(\sigma_{t}^{n-j+2}\right)\right)$ which is constant on $\sigma_{t}^{n-j+2}$ can as well be evaluated at an interior vertex $\sigma_{1}^{0} \subset \sigma^{n-j}$. Then our arguments for the interior case together with (8.12) imply that

$$
P_{\chi}\left(C^{\perp}\left(\sigma_{t}^{n-j+2}\right)\right)=O\left(\eta^{j-2}\right) .
$$

Since

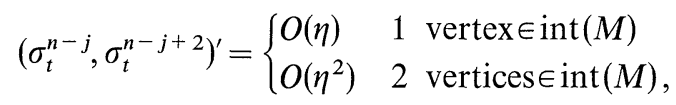

we see that (8.16) continues to hold in this case (and in particular, that those $\sigma^{n-j+2}$ with 2 vertices in the interior make a negligible contribution). The remainder of the construction of $\mathscr{H}^{j}$ and of the proof is a routine generalization of what was done in the interior case. Hence the details will be omitted. 
Fig. 8.1

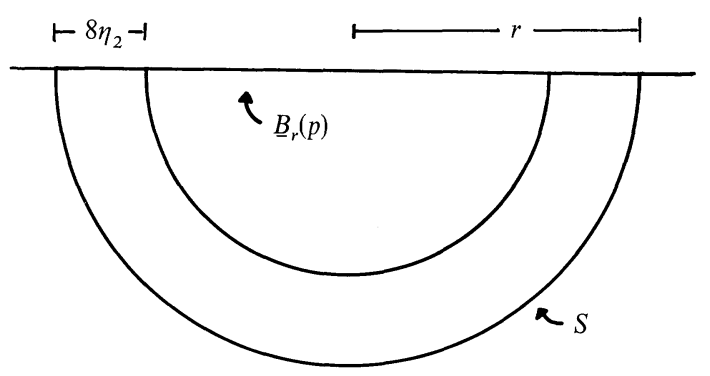

Proof of Lemma 8.4. The boundary of $B_{r}(p)$ can be written as $\underline{B}_{r}(p) \cup S$ as in Fig. 8.1. By a boundary version of Lemma 6.3 , proved by similar arguments, we can find $T_{3}, T_{4}$ which agree near $S$, and such that $T_{i}=T_{i+2}$ on $B_{r-8 \eta_{2}}(p)$. By Lemma 8.3 we can replace $T_{1}, T_{2}$ by $T_{3}, T_{4}$. Since $\tilde{T}_{3}, \tilde{T}_{4}$ agree except at interior points of $M^{+}$or $\partial M^{+}$, it follows that

$$
R_{\tilde{\eta}_{3}}^{j}+H_{\tilde{\eta}_{3}}^{j}=R_{\tilde{\eta}_{4}}^{j}+H_{\tilde{\eta}_{4}}^{j}
$$

By arguing as in the proof of (5.19) [see (6.17)-(6.26)], it follows that

$$
R_{\eta_{3}}^{j}+H_{\eta_{3}}^{j}=R_{\eta_{4}}^{j}+H_{\eta_{4}}^{j}+O(r)
$$

But $R_{\eta_{3}}^{j} \sim R_{\eta_{4}}^{j}$ by our result for the interior case. Since $T_{3}=T_{4}$ near $S$, the equality of the contributions to $H_{\eta_{3}}^{j}, H_{\eta_{4}}^{j}$ coming from $\underline{B}_{r}(p)$ follows. This suffices to complete the proof.

\section{Appendix 1. Local Calculations}

Recall that for $j=2, n=2$ the proof of Theorem 5.1 was based on a purely local calculation, (4.7). Moreover in this case we obtain an error which is $O(\eta)$. However, for $j \geqq 2, n>2$ we used a different argument which did not require complete knowledge of the polynomial $P_{j}$ of (5.13). We needed only the fact that $P_{j}$ (exists and) gives Lemma 5.5. But, for $j \geqq 2, n>2$ the error in Theorem 5.1 is only $O\left(\eta^{1 / 2}\right)$ for $U$ with smooth boundary. One purpose of this appendix is to bring into focus the difficulty in extending the local argument and improved error bounds to higher dimensional cases. We will concentrate on the case $j=2$ which is for the most part typical.

The choice of the particular polynomial $P_{2}$ can be viewed as depending on a convention concerning the value of the dihedral angle $\left(\sigma^{n-2}, \sigma^{n}\right)$ along $\sigma^{n-2} \subset \sigma^{n} \subset M^{n}$. Observe that $\left(\sigma^{n-2}, \sigma^{n}\right)$ varies over $\sigma^{n-2}$ and the precise pointwise value depends on the explicit choice of $(n-1)$-faces $\sigma_{1}^{n-1}, \sigma_{2}^{n-1} \subset \sigma^{n}$, with $\sigma_{1}^{n-1} \cap \sigma_{2}^{n-1}=\sigma^{n-2}$. However, at the vertices $\sigma_{i}^{0}$ of $\sigma^{n-2}$, the tangent planes to $\sigma_{1}^{n-1}, \sigma_{2}^{n-1}$ are determined by the tangent vectors to the (geodesic) 1-skeleton of $\sigma^{n}$. Thus, dihedral angles $\left(\sigma^{n-2}, \sigma^{n}\right)_{\sigma_{i}^{0}}$ are well defined at the vertices $\sigma_{i}^{0} \subset \sigma^{n-2}$. In the definition of $P_{i}$ we have, in effect, put:

$$
\left(\sigma^{n-2}, \sigma^{n}\right)=\frac{1}{n-1} \sum_{\sigma_{\imath}^{0}<\sigma^{n-2}}\left(\sigma^{n-2}, \sigma^{n}\right)_{\sigma_{\imath}^{0}}
$$


(compare the discussion preceding Proposition 5.2). Simple examples show that typically

$$
\left|\left(\sigma^{n-2}, \sigma^{n}\right)_{\sigma_{i}^{0}}-\left(\sigma^{n-2}, \sigma^{n}\right)_{\sigma_{j}^{0}}\right|=O\left(\eta^{2}\right) .
$$

Since also $\left(\sigma^{n-2}, \sigma^{n}\right)_{\sigma_{i}^{0}}-\left(\sigma^{n-2}, \sigma^{n}\right)_{\eta}=O\left(\eta^{2}\right)$, this variation is not negligible in the limit [a variation which is $O\left(\eta^{2}\right)$ is qualitatively consistent with a choice of higher dimensional faces $\sigma^{i}$ having principal curvatures which are $\left.O(\eta)\right]$.

The point of the preceding section is the following: Finding a local proof of Theorem 5.1 [yielding an error $O(\eta)$ ] is equivalent to "correctly" defining the dihedral angle $\left(\sigma^{n-2}, \sigma^{n}\right)$ (or what is essentially the same thing, making the correct choice for the faces $\sigma^{n-1}$ ). Unfortunately we don't know how to do this (see below for a more precise discussion).

We now give the explicit formula for $P_{2}$ (the derivation is at the end of this appendix). Fix $\sigma^{0} \subset \sigma^{n-2} \subset M^{n}$. Let $v_{1}, \ldots, v_{n} \subset M_{\sigma^{0}}$ be the tangent vectors to the edges of the lift $\exp _{\sigma^{0}}^{-1}\left(\sigma^{n}\right),\left\|v_{j}\left|\|=l_{j}=\right| \sigma_{j}^{1} \mid\right.$. Thus the $i$-faces $\sigma_{(j)}^{i}$, containing the origin are identified with

$$
\frac{1}{i !} v_{1} \wedge \ldots \wedge \hat{v}_{j_{1}} \wedge \ldots \wedge \hat{v}_{j_{n-i}} \ldots v_{n} .
$$

Following the notation of Sect. 2, the $(n-1)$-faces are denoted by $\frac{1}{(n-1) !} w_{j}$ and we assume that say [see (2.20)]

$$
\sigma^{n-2}=\frac{1}{(n-1) !} w_{i} \cap \frac{1}{(n-1) !} w_{j} .
$$

Then the contribution to $R^{2}\left(M_{\eta}^{n}\right)$ due to $\sigma^{n-2}$ is

$$
\begin{gathered}
\sum_{\sigma^{n} \supset \sigma^{n-2}} \frac{1}{3\left(n !\left|\sigma^{n}\right|\right)^{3}} \sum_{t, s}\left\{-\left\langle w_{i}, w_{s}\right\rangle\left\langle w_{j}, w_{t}\right\rangle\right. \\
+\frac{1}{2}\left\langle w_{i}, w_{j}\right\rangle\left\langle w_{j}, w_{s}\right\rangle \frac{\left\langle w_{j}, w_{t}\right\rangle}{\left\langle w_{j}, w_{j}\right\rangle} \\
\left.+\frac{1}{2}\left\langle w_{j}, w_{i}\right\rangle\left\langle w_{i}, w_{s}\right\rangle \frac{\left\langle w_{i}, w_{t}\right\rangle}{\left\langle w_{i}, w_{i}\right\rangle}\right\} \\
\cdot R\left(v_{t}, v_{s}, v_{s}, v_{t}\right)+O\left(\eta^{n+1}\right)
\end{gathered}
$$

$(1 \leqq i, j, t, s \leqq n)$. For each fixed $\sigma^{n}$, the expression in (A1.5) depends on the choice of $\sigma^{0} \subset \sigma^{n}$, but the sum over $\sigma^{n}$ is independent of this choice.

Although (A1.5) is linear in curvature, we know of no region $U$ for which it can be used to show directly that $R_{\eta}^{2}(U) \sim R^{2}(U)$. For example, if $U=s t\left(\sigma^{0}\right), R^{2}(U)$ can be obtained from (A1.5) by summing over $i, j$. This gives

$$
\sum_{\sigma^{n}>\sigma^{0}} \frac{1}{3\left(n !\left|\sigma^{n}\right|\right)^{3}} \sum_{\substack{1 \leq t, s \leqq n \\ 0 \leqq k \leqq n}}\left\langle w_{t}, w_{k}\right\rangle\left\langle w_{s}, w_{k}\right\rangle \frac{\left\langle w_{0}, w_{k}\right\rangle}{\left\langle w_{k}, w_{k}\right\rangle} R\left(v_{s}, v_{t}, v_{t}, v_{s}\right\rangle .
$$

Although (A1.6) is also not obviously related to the scalar curvature, as mentioned above, we could still succeed in finding a local proof of Theorem 5.1 by 
"correctly" redefining the dihedral angle $\left(\sigma^{n-2}, \sigma^{n}\right)$. More precisely it would suffice to have for each $\sigma^{n-2} C \sigma^{n}$, a number $\Theta\left(\sigma^{n-2}, \sigma^{n}\right)$, such that

A) $\Theta\left(\sigma^{n-2}, \sigma^{n}\right)=O\left(\eta^{2}\right)$.

For fixed $\sigma^{n-2}$,

B) $\sum_{\sigma^{n} \supset \sigma^{n-2}} \Theta\left(\sigma^{n-2}, \sigma^{n}\right)=0$.

C) The sum of $\Theta\left(\sigma^{n-2}, \sigma^{n}\right)$ and the expression inside the first summation in

$$
\begin{gathered}
\text { (A1.6) is equal to [see (A1.14) below] } \\
\frac{3}{2 n !} \sum_{1 \leqq i, j, k, l \leqq n}\left\langle w_{i} \wedge w_{j}, w_{k} \wedge w_{l}\right\rangle R\left(v_{i}, v_{j}, v_{l}, v_{k}\right) \text {. }
\end{gathered}
$$

As mentioned above, we don't know how to find $\Theta\left(\sigma^{n-2}, \sigma^{n}\right)$ satisfying A)-C). We point out that in view of (A1.5) Theorem 5.1 has a somewhat unexpected equivalent reformulation. To explain this, we first consider an arbitrary vector space $F^{n}$ with inner product $g$. Let $f^{*} \in\left(F^{m}\right)^{*}$ be the dual of $f$ with respect to $g$. Let $I: F^{m} \rightarrow F^{m}$ be the identity transformation and $f_{1}, \ldots, f_{N} \in F^{m}$. Then it is easy to see that

$$
\operatorname{tr} B=\sum_{i=1}^{N} B\left(f_{i}, f_{i}\right)
$$

for all symmetric bilinear forms $B$, if and only if

$$
\sum_{i=1}^{N} f_{i} \otimes f_{i}^{*}=I .
$$

Now let $F^{n}=\Lambda^{2}\left(E^{n}\right)$ and $B=R$ [the curvature tensor viewed as a symmetric bilinear form on $\Lambda^{2}\left(E^{n}\right)$ ]. The equivalence of (A1.8) and (A1.9) allows us to combine Theorem 5.1 with (A1.6) ( and rescale). This gives

Theorem A1.1. Let $T$ be any triangulation of $E^{n}$ of mesh 1 and fatness $\geqq \Theta$. Then

$$
\begin{aligned}
\lim _{r \rightarrow \infty} & \frac{1}{\left|B_{r}(0)\right|} \sum_{\sigma^{0} \subset \sigma^{n} \subset B_{r}(0)} \frac{1}{3\left(n !\left|\sigma^{n}\right|\right)^{3}} \\
& \cdot \lim _{\substack{1 \leqq s, t \leqq n \\
0 \leqq k \leqq n}}\left\langle w_{t}, w_{k}\right\rangle\left\langle w_{s}, w_{k}\right\rangle \frac{\left\langle w_{0}, w_{k}\right\rangle}{\left\langle w_{k}, w_{k}\right\rangle}\left(v_{s} \wedge v_{t}\right) \otimes\left(v_{s} \wedge v_{t}\right)^{*} \\
& =I+O\left(r^{-1}\right) .
\end{aligned}
$$

Consider in particular, a triangulation $T$ which is periodic, i.e., invariant under a lattice $\mathbb{Z}^{n} \subset E^{n}$. Then the quantity under the summation is invariant under $\mathbb{Z}^{n}$ and we obtain the following exact formula for the (finite) sum over $\Sigma^{2} / \mathbb{Z}^{n}$ (where $\Sigma^{2}$ is the 2-skeleton).

Corollary A1.2. Let $T$ be as in Theorem A1.1 and invariant under a lattice $\mathbb{Z}^{n}$. Then

$$
\begin{aligned}
& \frac{1}{\left|E^{n} / \mathbb{Z}^{n}\right|} \sum_{\Sigma^{n} / \mathbb{Z}^{n}} \frac{1}{3\left(n !\left|\sigma^{n}\right|\right)^{3}}\left\langle w_{t}, w_{k}\right\rangle\left\langle w_{s}, w_{k}\right\rangle \frac{\left\langle w_{0}, w_{k}\right\rangle}{\left\langle w_{k}, w_{k}\right\rangle} \\
& \quad \cdot\left(v_{s} \wedge v_{t}\right) \otimes\left(v_{s} \wedge v_{t}\right)^{*} \\
& \quad=I .
\end{aligned}
$$


A simple triangulation to which Theorem A1.1 applies is obtained by taking the barycentric subdivision of a paralellopiped as the basic unit. At an early stage of our work, we verified (A1.11) for this example by a straightforward but laborious computation.

We close this appendix with the derivation of (A1.5) (the derivation extends with trivial modifications to the case $j>2$ ).

It is apparent from (2.19) that if

$$
G=\left(\left\langle v_{i}, v_{j}\right\rangle\right),
$$

and

$$
W=\left(\left\langle w_{i}, w_{j}\right\rangle\right)
$$

then

$$
G \cdot W=\left\|v_{1} \wedge \ldots \wedge v_{n}\right\|^{2} I,
$$

since $W$ is the cofactor matrix of $G$. Set

$$
\begin{gathered}
\left\langle v_{i}, v_{j}\right\rangle=\left\|v_{i}\right\| \cdot\left\|v_{j}\right\| \cos \alpha_{i j}, \\
\left\|w_{i}\right\|=L_{i}, \\
L=\left(\begin{array}{cc}
L_{i} & 0 \\
& \ddots \\
0 & L_{n}
\end{array}\right), \\
X=\left(\cos \Theta_{i j}\right),
\end{gathered}
$$

where $\cos \Theta_{i i}=1$,

$$
s=\left\|v_{1} \wedge \ldots \wedge v_{n}\right\|^{2} .
$$

Now suppose that, $v_{1}, \ldots, v_{n}$ depend smoothly on a parameter $t$. We must calculate the derivatives $\Theta_{i j}^{\prime}$ in terms of the $\alpha_{i j}^{\prime}$. If we define the matrix $P$ by

$$
P=s^{1 / 2} \cdot\left(\left\|v_{1} \wedge \ldots \wedge \hat{v}_{i} \ldots \hat{v}_{j} \ldots v_{n}\right\| \Theta_{i j}^{\prime}\right)
$$

then by (A1.20), we also have

$$
P=\left(\left\|w_{i}\right\| \cdot\left\|w_{j}\right\| \sin \Theta_{i j} \Theta_{i j}^{\prime}\right)
$$

It will suffice to give a formula for $P$. To this end, differentiate (A1.14) to get

$$
W^{\prime} G+W G^{\prime}=s^{\prime} I \text {. }
$$

Multiplying on the right by $\frac{1}{S} W=G^{-1}$, gives

$$
W^{\prime}=\frac{-1}{s} W G^{\prime} W+\frac{s^{\prime}}{s} W .
$$

Also, since

$$
W=L X L
$$


we have

$$
W^{\prime}=L^{\prime} X L+L X^{\prime} L+L X L^{\prime}=L^{\prime} L^{-1} W+P+W L^{-1} L^{\prime} .
$$

Equating the diagonal terms, in (A1.23) and (A1.25) gives

$$
\left[\frac{1}{s} W G^{\prime} W\right]_{\mathrm{diag}}+\left[\frac{s^{\prime}}{s} W\right]_{\mathrm{diag}}=2 L^{\prime} L=2 L L^{\prime},
$$

where we have used $[P]_{\text {diag }}=0$. Then

$$
\begin{aligned}
L^{-1} L^{\prime}= & L^{\prime} L^{-1}=\frac{1}{2} L^{-2}\left[-\frac{1}{s} W G^{\prime} W\right]_{\mathrm{diag}} \\
& +\frac{1}{2} L^{-2}\left[\frac{s^{\prime}}{s} W\right]_{\mathrm{diag}} \\
= & \frac{1}{2} L^{-2}\left[\frac{-1}{s} W G^{\prime} W\right]_{\mathrm{diag}} \\
& +\frac{1}{2} \frac{s^{\prime}}{s} I \\
= & \frac{1}{2}\left[-\frac{1}{s} W G^{\prime} W\right]_{\mathrm{diag}} L^{-2} \\
& +\frac{1}{2} \frac{s^{\prime}}{s} I .
\end{aligned}
$$

By (A1.25)

$$
P=W^{\prime}-L^{\prime} L^{-1} W-W L^{-1} L^{\prime} .
$$

Substituting (A1.23), (A1.27) into (A1.28) gives the desired formula

$$
P=\frac{-1}{s} W G^{\prime} W+\frac{1}{2} W\left[\frac{1}{s} W G^{\prime} W\right]_{\mathrm{diag}} L^{-2}+\frac{1}{2} L^{-2}\left[\frac{1}{s} W G^{\prime} W\right]_{\mathrm{diag}} W
$$

\section{Appendix 2. Variable Curvature and Dihedral Angles}

In this appendix we give a proof of estimate (4.7) for the case of nonconstant curvature. It is obtained from the following estimate (A2.1) by a standard argument, the details of which will be omitted.

Let $\tau_{s}$ denote the plane section spanned by $\gamma_{i}^{\prime}(s)$ and $T$, the radial vector in normal coordinates. Let $P\left(\tau_{s}\right)$ denote its parallel translated to the origin along the radial geodesic to $\gamma_{i}(s)$.

Then for some universal constant $c$, the angle $\Varangle\left(P\left(\tau_{s}\right), \sigma\right)$ formed by $P\left(\tau_{s}\right)$ and $\sigma$ satisfies

$$
\left|\Varangle\left(P\left(\tau_{s}\right), \sigma\right)\right| \leqq c t .
$$

The estimate (A2.1) is, in turn, implied by

$$
\left\|\nabla_{\frac{\partial}{\partial x_{i}}} \frac{\partial}{\partial x_{j}}\right\| \leqq c t, \quad 1 \leqq i, j \leqq n,
$$


together with (the lower half of) the bound

$$
1-c t^{2} \leqq\left\|\frac{\partial}{\partial x_{i}}\right\| \leqq 1+c t^{2}
$$

for the normal coordinate field $\frac{\partial}{\partial x_{k}}$. [Estimate (A2.3) is implied by the RauchComparison theorem, see [CE], but we will give an elementary, self-contained derivation.]

To prove (A2.2), (A2.3) at a point $q$, it is convenient to choose normal coordinates, such that $q$ lies on the $x_{1}$-axis. Let the coordinates $\left(r, \Theta_{j}\right)(2 \leqq j \leqq n)$ be defined by

$$
\begin{aligned}
x_{1} & =r, \\
x_{j} & =r \tan \Theta_{j}, \quad j=2, \ldots, n .
\end{aligned}
$$

Note that $r$ is not the usual polar coordinate except along the $x_{1}$-axis. Then (A2.2), (A2.3) are equivalent to

$$
\begin{gathered}
r\left(1-c r^{2}\right) \leqq\left\|\frac{\partial}{\partial \Theta_{i}}\right\| \leqq r\left(1+c r^{2}\right), \\
\left(1-c r^{2}\right) \leqq\left\|\nabla_{T} \frac{\partial}{\partial \Theta_{i}}\right\| \leqq\left(1+c r^{2}\right), \\
\left\|\nabla_{T} \frac{\partial}{\partial x_{i}}\right\| \leqq c r, \\
\left\|\nabla_{\frac{\partial}{\partial \Theta_{\imath}}} \frac{\partial}{\partial \Theta_{j}}\right\| \leqq c r^{3}, \quad 2 \leqq i, j \leqq n
\end{gathered}
$$

[see (A2.23) for the connection between (A2.2) and (A2.8)] which we now will establish. Note that the restriction of $\frac{\partial}{\partial \Theta_{i}}$ to the $x_{1}$-axis is a Jacobi field. Hence along the $x_{1}$-axis we have the Jacobi equations

$$
\nabla_{T} \nabla_{T} \frac{\partial}{\partial \Theta_{i}}=R\left(T, \frac{\partial}{\partial \Theta_{i}}\right) T
$$

with the initial conditions

$$
\left.\frac{\partial}{\partial \Theta_{i}}\right|_{r=0}=0,\left.\quad \nabla_{T} \frac{\partial}{\partial \Theta_{i}}\right|_{r=0}=\left.\frac{\partial}{\partial x_{i}}\right|_{r=0} .
$$

We will use Taylor's formula in the form

$$
f(r)=\sum_{k=0}^{n-1} \frac{r^{k}}{k !} f^{(k)}(0)+\frac{1}{(n-1) !} \int_{0}^{r}(r-s)^{n-1} f^{(n)}(s) d s .
$$


As above, let $P$ denote parallel translation to the origin along the $x_{1}$-axis. Then by (A2.9)-(A2.11)

$$
\begin{aligned}
P\left(\frac{\partial}{\partial \Theta_{i}}\right) & =r \frac{\partial}{\partial x_{i}}+\int_{0}^{r}(r-s) P^{\prime \prime}\left(\frac{\partial}{\partial \Theta_{i}}\right) d s \\
& =r \frac{\partial}{\partial x_{i}}+\int_{0}^{r}(r-s) P\left(\nabla_{T} \nabla_{T} \frac{\partial}{\partial \Theta_{i}}\right) d s \\
& =r \frac{\partial}{\partial x_{i}}+\int_{0}^{r}(r-s) P\left(R\left(T, \frac{\partial}{\partial \Theta_{i}}\right) T\right) d s
\end{aligned}
$$

Since $P$ is an isometry, (A2.12) gives

$$
\left\|\frac{\partial}{\partial \Theta_{i}}\right\| \leqq r+c^{\prime} \int_{0}^{r}(r-s)\left\|\frac{\partial}{\partial \Theta_{i}}\right\| d s .
$$

Let $z(r)=\sup _{0 \leqq s \leqq r}\left\|\frac{\partial}{\partial \Theta_{i}}\right\|$. Then upon integration, estimate (A2.13) leads to

$$
z(r) \leqq r+c^{\prime} z(r) r^{2}
$$

and therefore

$$
\left\|\frac{\partial}{\partial \Theta_{i}}\right\| \leqq z(r) \leqq r\left(1-c^{\prime} r^{2}\right)^{-1} \leqq r+c r^{3} .
$$

To obtain the lower bound, we estimate (A2.12) as

$$
\left\|\frac{\partial}{\partial \Theta_{i}}\right\| \geqq r-c^{\prime} \int_{0}^{r}(r-s)\left\|\frac{\partial}{\partial \Theta_{i}}\right\| d s,
$$

and insert the upper bound (A2.15) on the right hand side. To prove (A2.6), we write

$$
\begin{aligned}
P\left(\nabla_{T} \frac{\partial}{\partial \Theta_{i}}\right)-\frac{\partial}{\partial x_{i}} & =\int_{0}^{r} P^{\prime}\left(\nabla_{T} \frac{\partial}{\partial \Theta_{i}}\right) d s \\
& =\int_{0}^{r} P\left(\nabla_{T} \nabla_{T} \frac{\partial}{\partial \Theta_{i}}\right) d s \\
& =\int_{0}^{r} P\left(R\left(T, \frac{\partial}{\partial \Theta_{i}}\right) T\right) d s .
\end{aligned}
$$

Hence

$$
\left\|P\left(\nabla_{T} \frac{\partial}{\partial \Theta_{i}}\right)-\frac{\partial}{\partial x_{i}}\right\| \leqq c^{\prime} \int_{0}^{r} s d s \leqq c r^{2},
$$

from which (A2.6) follows. Next, along the $x_{1}$-axis,

$$
\nabla_{T} \frac{\partial}{\partial x_{i}}=-\frac{1}{r^{2}} \frac{\partial}{\partial \Theta_{i}}+\frac{1}{r} \nabla_{T} \frac{\partial}{\partial \Theta_{i}} .
$$


Combining (A2.12) and (A2.17) (or equivalently, performing a partial integration), we obtain

$$
P\left(\nabla_{T} \frac{\partial}{\partial x_{i}}\right)=\frac{1}{r^{2}} \int_{0}^{r} s P\left(R\left(T, \frac{\partial}{\partial \Theta_{i}}\right) T\right) d s,
$$

from which (A2.7) follows, if we again use the upper bound of (A2.5). To prove the final estimate (A2.8), we use again the Jacobi equation

$$
\begin{aligned}
\nabla_{T} \nabla_{T} \nabla_{\frac{\partial}{\partial \Theta_{i}}} \frac{\partial}{\partial \Theta_{j}} & =\nabla_{T}\left(R\left(T, \frac{\partial}{\partial \Theta_{i}}\right) \frac{\partial}{\partial \Theta_{j}}\right)+\nabla_{T} \nabla_{\frac{\partial}{\partial \Theta_{i}}} \nabla_{T} \frac{\partial}{\partial \Theta_{j}} \\
& =\nabla_{T}\left(R\left(T, \frac{\partial}{\partial \Theta_{i}}\right) \frac{\partial}{\partial \Theta_{j}}\right)+R\left(T, \frac{\partial}{\partial \Theta_{i}}\right) \nabla_{T} \frac{\partial}{\partial \Theta_{j}}+\nabla_{\frac{\partial}{\partial \Theta_{i}}}\left(R\left(T, \frac{\partial}{\partial \Theta_{j}}\right) T\right) .
\end{aligned}
$$

The relation $\nabla_{T} \frac{\partial}{\partial \Theta_{i}}=\nabla_{\frac{c}{\partial \Theta_{\imath}}} T$, the upper bound in (A2.5) and (A2.6) then combine with (A2.21) to give

$$
\left\|\nabla_{T} \nabla_{T} \nabla_{\frac{\partial}{\partial \Theta_{i}}} \frac{\partial}{\partial \Theta_{j}}\right\| \leqq c^{\prime}\left(r+\left\|\nabla_{\frac{\partial}{\partial \Theta_{i}}} \frac{\partial}{\partial \Theta_{j}}\right\|\right) .
$$

We employ this estimate as follows. Since along the $x_{1}$-axis

$$
\nabla_{\frac{\partial}{\partial x_{i}}} \frac{\partial}{\partial x_{j}}=\frac{1}{r^{2}} \nabla_{\frac{\partial}{\partial \Theta_{i}}} \frac{\partial}{\partial \Theta_{j}}, \quad 2 \leqq i, j \leqq n,
$$

we have

$$
\left.\nabla_{\frac{\partial}{\partial \Theta_{\imath}}} \frac{\partial}{\partial \Theta_{j}}\right|_{r=0}=\left.\nabla_{T} \nabla_{\frac{\partial}{\partial \Theta_{i}}} \frac{\partial}{\partial \Theta_{j}}\right|_{r=0}=0, \quad 2 \leqq i, j \leqq n .
$$

Therefore

$$
\begin{aligned}
P\left(\nabla_{\frac{\partial}{\partial \Theta_{\imath}}} \frac{\partial}{\partial \Theta_{j}}\right) & =\int_{0}^{r}(r-s) P^{\prime \prime}\left(\nabla_{\frac{\partial}{\partial \Theta_{i}}} \frac{\partial}{\partial \Theta_{j}}\right) d s \\
& =\int_{0}^{r}(r-s) P\left(\nabla_{T} \nabla_{T} \nabla_{\frac{\partial}{\partial \Theta_{j}}} \frac{\partial}{\partial \Theta_{j}}\right) d s
\end{aligned}
$$

Let now $z(r)=\sup _{0 \leqq s \leqq r}\left\|\nabla_{\frac{\partial}{\partial \Theta_{i}}} \frac{\partial}{\partial \Theta_{j}}\right\|$. Then the relations (A2.22) and (A2.25) combine to give

$$
z(r) \leqq c^{\prime}\left(\frac{r^{3}}{6}+\frac{r^{2}}{2} z(r)\right)
$$

from which (A2.8) follows. The equivalence of the estimates (A2.2)-(A2.3) and (A2.5)-(A2.8) now follows easily using (A2.23). 


\section{Appendix 3. Riemannian Metric on the Space of Piecewise Flat Structures}

In this appendix we describe some additional properties of p.l. spaces. They come into play if one attempts to quantize gravity by using these spaces as an intermediate step in arriving at a continuum theory (see e.g. [CMS1]). We will begin by characterizing those p.l. spaces which have the same underlying simplicial complex. Then mimicking the procedure which makes the set of all Riemannian metrics on a manifold an infinite dimensional Riemannian space (see e.g. [E]), we shall make this set a noncompact Riemannian space. This leads to a volume form, allowing one to consider "functional integrals" and statistical mechanics of p.1. spaces. The construction, however, has a certain nonuniqueness which can be thought of in terms of an analog of the conformal group.

As in Sect. 3, let $\sigma^{n}$ be a $n$-simplex in $E^{k}$ with vertices given by the vectors $v_{0}=0$, $v_{1}, \ldots, v_{n}$. The matrix

$$
g_{i j}=\left\langle v_{i}, v_{j}\right\rangle \quad(1 \leqq i, j \leqq n)
$$

is positive definite and

$$
\operatorname{det} g_{i j}=\left(\left|\sigma^{n}\right| n !\right)^{2}
$$

In terms of the edge lengths

$$
l(i, j)=\left\|v_{i}-v_{j}\right\| \quad(0 \leqq i, j \leqq n),
$$

we have

$$
g_{i j}=\frac{1}{2}\left(l^{2}(0, i)+l^{2}(0, j)-l^{2}(i, j)\right) .
$$

Conversely, given a function $l(\cdot) \geqq 0$ on the edges of the ordered standard $n$-simplex ${ }^{s} \sigma^{n}$, an easy argument by induction shows that the values of $l(\cdot)$ form the edge lengths of a simplex $\sigma^{n}$ in $E^{n}$ if and only if $g_{i j}$ as defined by (A3.4) is positive definite. By a well known theorem in linear algebra this holds if and only if

$$
\left(\operatorname{det} g_{i j}\right)_{i, j \in I}>0
$$

for every nonempty subset $I$ of $(1, \ldots, n)$. In that case, by (A3.2), the left hand side of (A3.5) is $(|I| !)^{2}$ times the volume squared of the $|I|$-simplex formed by $v_{0}$ and $v_{i}$ $(i \in I)$. The polynomial inequalities (A3.5) in the edgelengths form the higher dimensional analogues of the triangle inequalities.

We recall that the set of all positive definite $n \times n$ matrices form a symmetric space $P$. If $a$ and $a^{\prime}$ denote two elements of the tangent space at $g=\left\{g_{i j}\right\}$, i.e. $a$ and $a^{\prime}$ are real symmetric matrices, we define

$$
G_{g}\left(a, a^{\prime}\right)=\sum_{i, j, k, l} g^{i j} a_{j k} g^{k l} a_{l i}^{\prime}\left(\operatorname{det} g_{i j}\right)^{1 / 2}
$$

to be the metric on the tangent space at $g$. Here $g^{i j}$ is the inverse of $g_{i j}$. [The factor $\left(\operatorname{det} g_{i j}\right)^{1 / 2}$ was carelessly omitted in [CMS1]. It improves the short distance behavior of the theory.]

Now let $\hat{\zeta}$ be the space of ordered $n$-simplices with given edge lengths and let $\sigma^{n} \in \hat{\zeta}$. Define an isomorphism from the isometry classes of such $\sigma^{n}$ into $P$ by (A3.4). The pull back of the metric to $\hat{\zeta}$ via this isomorphism gives a metric which is 
independent of the ordering. Thus it makes the set of all unordered $n$-simplices a Riemannian space which we denote by $\zeta$. Now fix the combinatorial structure $K^{n}$ and let the metric structure $\left(K^{n}, l\right)$ vary. Assume each simplex is contained in a $n$-simplex. There is a natural embedding

$$
\left\{\left(K^{n}, l\right)\right\} \rightarrow \prod_{\sigma^{n} \in K}\left(\lambda\left(\sigma^{n}\right) \zeta\right)
$$

Here $\lambda\left(\sigma^{n}\right)>0$ are arbitrary and $\lambda \zeta$ denotes the metric $\zeta$ scaled by $\lambda>0$. The Riemannian metric $\zeta\left(K^{n}, \lambda(\cdot)\right)$ in the collection of all $\left\{\left(K^{n}, l\right)\right\}$ is induced from this embedding, making it into a finite dimensional Riemannian space.

Due to the arbitrariness of the $\lambda\left(\sigma^{n}\right)$, our construction is not unique. The nonuniqueness is best described in terms of the conformal group, defined in this context to be the set of all positive valued functions $\varrho\left(\sigma^{n}\right)$ on the $n$-simplices of $K^{n}$. This group obviously acts as an effective, transitive transformation group on the set of all such metrics $\zeta\left(K^{n}, \lambda(\cdot)\right)$ by

$$
\varrho(\cdot): \zeta\left(K^{n}, \lambda(\cdot)\right) \rightarrow \zeta\left(K^{n}, \varrho(\cdot) \lambda(\cdot)\right) .
$$

Acknowledgements. At various stages of our work, we had the opportunity to profit from discussions with J. Fröhlich, M. Gromov, H. Leutwyler, J. Milnor, and P. Wintgen (†). One of us (R.S.) would like to thank Prof. C. N. Yang for his hospitality at the Institute for Theoretical Physics at SUNY, Stony Brook.

\section{References}

[AW] Allendoerfer, C.B., Weil, A.: The Gauss-Bonnet theorem for Riemannian polyhedra. Trans. Am. Math. Soc. 53, 101-129 (1943)

[B] Banchoff, T.: Critical points and curvature for embedded polyhedra. J. Diff. Geom. 1, 245-256 (1967)

[Br] Brin, I.A.: Gauss-Bonnet theorems for polyhedrons. Uspekhi Mat. Nauk III, 226-227 (1948) (in Russian)

[C] Cheeger, J.: Spectral geometry of singular Riemannian spaces. J. Diff. Geom. 18 (1983)

[CE] Cheeger, J., Ebin, D.G.: Comparison theorems in Riemannian geometry. Amsterdam: North-Holland 1975

[CMS1] Cheeger, J., Müller, W., Schrader, R.: In : Unified theories of elementary particles (Heisenberg Symposium 1981). Lecture Notes in Physics. Breitenlohner, P., Dürr, H.P. (eds.). Berlin, Heidelberg, New York: Springer 1982

[CMS2] Cheeger, J., Müller, W., Schrader, R.: In preparation

$[\mathrm{Ch}]$ Chern, S.S.: On the kinematic formula in integral geometry. J. Math. Mech. 16, 101-118 (1966)

[CW1] Collins, P.A., Williams, R.M.: Application of Regge calculus to the axially symmetric initialvalue problem in general relativity. Phys. Rev. D5, 1908-1912 (1972)

[CW2] Collins, P.A., Williams, R.M.: Dynamics of the Friedmann universe using Regge calculus. Phys. Rev. D7, 965-961 (1973)

[CW3] Collins, P.A., Williams, R.M.: Regge-calculus model for the Tolman universe. Phys. Rev. D 10, 3537-3538 (1974)

[D] Donnelly, H.: Heat equation and the volume of tubes. Invent. Math. 29, 239-243 (1975)

[E] Ebin, D.G.: In: Global analysis. Proceedings of Symposium in Pure Mathematics, Vol. XV. Providence, RI: American Mathematical Society 1970

[F] Freudenthal, H.: Simplizialzerlegung von beschränkter Flachheit. Ann. Math. 43, 580-582 (1942)

[Fr] Fröhlich, J.: IHES preprint 1981 (unpublished) 
[G1] Gilkey, P.B.: The index theorem and the heat equation. Boston: Publish or Perish 1974

[G2] Gilkey, P.B.: The boundary integrand in the formula for the signature and Euler characteristic of a Riemannian manifold with boundary. Adv. Math. 15, 334-360 (1975)

[HS] Hartle, J.B., Sorkin, R.: Boundary terms in the action for the Regge calculus. Gen. Rel. Grav. 13, 541-549 (1981)

[HP] Hasslacher, B., Perry, M.: Spin networks are simplicial quantum gravity. Phys. Lett. 103B, 21-24 (1981)

[H] Hawking, S.W.: In: General relativity, an Einstein centenary survey. Hawking, S.W., Israel, W. (eds.). Cambridge: Cambridge University Press 1979

[K] Kneser, H.: Der Simplexinhalt in der nichteuklidischen Geometrie. Deutsch. Math. 1, 337-340 (1936)

[L1] Lewis, S.M.: Two cosmological solutions of Regge calculus. Phys. Rev. D25, 306-312 (1982)

[L2] Lewis, S.M.: Maryland Thesis, Preprint (1982)

[McC] McCrory, C.: Stratified general position. In: Lecture Notes in Mathematics, Vol. 664. Berlin, Heidelberg, New York: Springer 1978

[Mc] McMullen, P.: Non-linear angle-sum relations for polyhedral cones and polytopes. Math. Proc. Camb. Philos. Soc. 78, 247-261 (1975)

[Mi] Minkowski, H.: In: Gesammelte Abhandlungen, Vol. II, pp. 131-229. New York: Chelsea Publ. 1967

[MTW] Misner, C.W., Thorn, K.S., Wheeler, J.A.: Gravitation. San Francisco: Freeman 1973

[Mu] Munkres, J.R.: Elementary differential topology. Princeton, NJ : Princeton University Press 1966

[PR] Ponzano, G., Regge, T.: In: Spectroscopic and group theoretical methods in physics. Bloch, F., Cohen, S.G., De Shalit, A., Sambursky, S., Talmi, I. (eds.). New York: Wiley 1968

[Ra] Rado, T.: Length and area. New York: American Mathematical Society 1948

[R] Regge, T.: General relativity without coordinates. Nuovo Cimento 19, 558-571 (1961)

[RW1] Roček, M., Williams, R.M.: Quantum Regge calculus. Phys. Lett. 104B, 31-37 (1981)

[RW2] Roček, M., Williams, R.M.: The quantization of Regge calculus. Preprint (1981)

[Sa] Santalo, L.A.: Integral geometry and geometric probability. London: Addison-Wesley 1976

[Sc1] Schläfli, L.: On the multiple integral $\int^{n} d x d y \ldots d x$, whose limits are $p_{1}=a_{1} x+b_{1} y$ $+\ldots+h_{1} z>0, p_{2}>0, \ldots, p_{n}>0$ and $x^{2}+y^{2}+\ldots+z^{2}<1$. Q. J. Pure Appl. Math. 2, 269-301 (1858)

[Sc2] Schläfli, L.: Über die Entwickelbarkeit des Quotienten zweier bestimmter Integrale von der Form $\int d x d y \ldots d z$. J. Reine Angew. Math. 67, 183-199 (1867)

[SO1] Sorkin, R.: Time-evolution problem in Regge calculus. Phys. Rev. D 12, 385-396 (1975)

[SO2] Sorkin, R. : The electromagnetic field on a simplicial net. J. Math. Phys. 16, 2432-2440 (1975)

[St] Steiner, J.: Jber preuss. Akad. Wiss. 114-118 (1840). In: Gesammelte Werke, Vol. II, pp. 171-177, New York: Chelsea 1971

[SW] Sulanke, R., Wintgen, P.: Differentialgeometrie und Faserbündel. Berlin: Deutscher Verlag der Wissenschaft 1972

[W] Warner, N.P.: The application of Regge calculus to quantum gravity and quantum field theory in a curved background. Proc. R. Soc. 383, 359-377 (1982)

[Wei] Weingarten, D.: Euclidean quantum gravity on a lattice. Nucl. Phys. B210, 229-249 (1982)

[Wey] Weyl, H.: On the volume of tubes. Am. J. Math. 61, 461-472 (1939)

[Whe] Wheeler, J.A.: In: Relativity, groups, and topology. deWitt, C., deWitt, B. (eds.). New York: Gordon and Breach 1964

[Whi] Whitney, H.: Geometric integration theory. Princeton, NJ : Princeton University Press 1971

[WE] Williams, R.M., Ellis, G.F.R.: Regge calculus and observations. I. Formalism and applications to radial motion and circular orbits. Gen. Rel. Grav. 13, 361-395 (1981)

[Wi] Wintgen, P. : Normal cycle and integral curvature for polyhedra in Riemannian manifolds. Colloquia Mathematica Societatis Janos Bolya, Budapest (1978)

[Wo] Wong, C.Y.: Application of Regge calculus to the Schwarzschild and Reissner-Nørdstrom geometries at the moment of time symmetry. J. Math. Phys. 12, 70-78 (1971)

Communicated by A. Jaffe

Received June 21, 1983; in revised form October 3, 1983 